\title{
Host Tau Genotype Specifically Regulates and Designs Tau Seeding and Spreading Following Intracerebral Injection of Identical Tau AD Inoculum
}

\section{Pol Andrés-Benito}

IDIBELL: Institut d'Investigacio Biomedica de Bellvitge

\section{Margarita Carmona}

IDIBELL: Institut d'Investigacio Biomedica de Bellvitge

\section{Mónica Jordán}

CIBERNED: Centro de Investigacion Biomedica en Red sobre Enfermedades Neurodegenerativas

\section{José Antonio del Rio}

IBEC: Institut de Bioenginyeria de Catalunya

Isidro Ferrer ( $\nabla$ 8082ifa@gmail.com )

Universitat de Barcelona https://orcid.org/0000-0001-9888-8754

\section{Research}

Keywords: host tau, seeding and spreading, 3Rtau and 4Rtau, P301S, hTau, Alzheimer's disease, tauopathies

Posted Date: October 27th, 2021

DOI: https://doi.org/10.21203/rs.3.rs-889543/v2

License: (c) (i) This work is licensed under a Creative Commons Attribution 4.0 International License. Read Full License 


\section{Abstract}

Background: Several studies have demonstrated the capacity for seeding and spreading of tau-enriched fractions of brain homogenates from $A D$ and other human and mouse tauopathies following intracerebral inoculation into transgenic mice bearing human tau or mutant human tau and into WT mice. However, little attention has been paid about the importance of host tau in tau seeding.

Methods: The brains of four adult murine genotypes expressing different forms of tau-WT (murine 4Rtau), P301S (human 4Rtau expressing the P301S mutation), hTau (homozygous transgenic mice knock-out for murine tau protein and heterozygous expressing human forms of 3Rtau and 4Rtau proteins), and $\mathrm{mtWt}$ (homozygous transgenic mice knock-out for murine tau protein)-were analyzed following unilateral hippocampal inoculation of sarkosyl-insoluble tau fractions from the same AD case.

Results: No tau deposits were identified in inoculated mtWT mice. Involvement of CA1 neurons was higher and that of oligodendrocytes lower in inoculated hTau when compared with inoculated WT and P301S mice. tau-P Ser422, PHF1, and MAP2-P immunoreactivity was moderate or weak in WT and P301S, but strong in inoculated hTau mice. p38-P and SAPK/JNK-P were observed in recruited phospho-

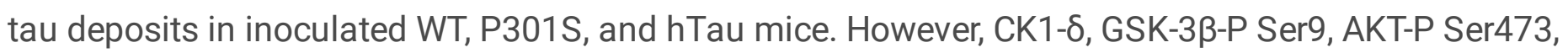
PKAa/ $\beta-P$ Tyr197, and CLK1 were identified in neurons with tau deposits only in inoculated hTau. Finally, 3Rtau deposits predominated in inoculated WT and P301S, and 4Rtau deposits in hTau transgenic mice.

Conclusions: Our results reveal that a) host tau is mandatory for tau seeding and spreading following tau inoculation; b) tau seeding and spreading is characterized by major genotype-dependent biochemical changes linked to post-translational tau modifications including tau phosphorylation and tau nitration at different sites, c) it is accompanied by genotype-dependent activation of various kinases thus pointing to a complex molecular response in the receptive host cells; d) tau seeding and spreading is accompanied by modifications in tau splicing with variable expression of new 3Rtau and 4Rtau isoforms; e) selective regional and cellular vulnerabilities, and different molecular compositions of the deposits are dependent on the host tau genotypes injected with identical AD tau inoculum.

\section{Background}

Neurodegenerative diseases with abnormal protein aggregates are characterized neuropathologically by the deposition of protein aggregates mainly resulting from aberrant post-translational modifications of primary constitutive elements of the nervous system. The term tauopathy covers heterogeneous diseases having in common the neuronal and glial deposition of abnormally phosphorylated species of tau protein, usually accompanied by other post-translational modifications in tau [1-3].

Selective neuronal and glial vulnerability and progression are determinants in neurodegenerative diseases with abnormal protein aggregates [4, 5]. Several in vivo studies demonstrate the capacity for seeding and spreading of tau following intracerebral inoculation of transgenic mice expressing $4 \mathrm{R}$ human tau or mutant human tau. Tau seeding and spreading is produced following the inoculation of synthetic tau 
fibrils $[6,7]$ or inoculation of fibrillar-enriched fractions from human and mouse brain homogenates of tauopathies, including Alzheimer's disease (AD), only tangle dementia, progressive supranuclear palsy (PSP), corticobasal degeneration (CBD), argyrophilic grain disease (AGD), as well as in tau transgenic mice [8-12]. Tau seeding and spreading also occurs following intracerebral inoculation of similar tau aggregates in wild type mice (WT) expressing murine tau [9, 13-15]. In addition to neurons, deposits also occur in glial cells, and the morphology of glial inclusions appears to mimic the glial aggregates of the corresponding human tauopathies in inoculated transgenic mice expressing human tau or mutant human tau $[9-12,16]$, and in WT mice [14-16].

It is widely accepted that tau strains are behind the different phenotypes and progression of human tauopathies [5, 17-20]. Abnormal tau may also have different effects on the host; for example, P301L seeds are uniquely modified by post-translational modifications within the microtubule-binding region resulting from histone deacetylase 6 (HDAC6) inhibition and tau auto-acetylation; HDAC6 inhibition results in accelerated tau aggregation [21].

However, our previous studies of tau seeding and spreading in WT mice show similar cellular vulnerability despite the origins of the inocula. Inoculation of sarkosyl-insoluble fractions of brain homogenates from human tauopathies, including $A D$, primary age-related tauopathy (PART), aging-related tau astrogliopathy (ARTAG), PSP, AGD, Pick's disease (PiD), frontotemporal lobar degeneration linked to MAPT P301L mutation, and globular glial tauopathy (GGT) reproduces a tauopathy, but inoculated mice do not express the markers of specific tauopathies [22-26]. Pick bodies, thorn-shaped astrocytes, tufted astrocytes, astrocytic plaques, and glial globular inclusions are not observed, but coiled bodies in oligodendrocytes are constant even following inoculation of $A D$ and PART homogenates, diseases in which tau deposits do not occur in oligodendroglia [24]. Conversely, intracerebral inoculation of brain homogenates of pure ARTAG cases, which do not have tau deposits in neurons, induces neuronal tau seeding and spreading in addition to oligodendroglial inclusions [22]. Moreover, of inoculated homogenates, despite their primary tau composition, 3Rtau plus 4Rtau (AD, PART), only 4Rtau (ARTAG, PSP, GGT, FTLD-P301L) and 3Rtau (PiD), produce 3Rtau and 4Rtau deposits in WT mice [22-26]. These observations suggest that not only the origin of the donor tau but also the characteristics of the host tau are relevant in tau seeding and spreading [26].

Following this line of thinking, this study analyzes the characteristics of tau seeding and spreading following intrahippocampal inoculation of insoluble tau (sarkosyl-insoluble fractions) from the same $A D$ case to four murine models expressing different forms of tau: WT (4Rtau murine in adults), P301S (human 4Rtau expressing the P301S mutation), hTau (homozygous transgenic mice knock-out for murine tau protein and heterozygous expressing human forms of 3Rtau and 4Rtau proteins), and $\mathrm{mtWt}$ (homozygous transgenic mice knock-out for murine tau protein). Our endeavor is to gain understanding about the different regulation and modelling of tau seeding and spreading depending on the characteristics of host tau.

\section{Methods}




\section{Brain samples}

Brain samples of the hippocampus were obtained from the Institute of Neuropathology Brain Bank, Bellvitge University Hospital, following the guidelines of the Spanish legislation on this matter (Real Decreto Biobancos 1716/2011), and the approval of the local ethics committee of the Bellvitge University Hospital (Hospitalet de Llobregat, Barcelona, Spain). The agonal state was short with no evidence of acidosis or prolonged hypoxia; the $\mathrm{pH}$ of the brains was between 6.8 and 7. At the time of autopsy, one hemisphere was fixed in paraformaldehyde for no less than 3 weeks, and selected brain sections were embedded in paraffin; de-waxed paraffin sections, 4 microns thick, were processed with neuropathological and immunohistochemical methods as detailed elsewhere [27]. The other hemisphere was cut into coronal sections $1 \mathrm{~cm}$ thick, and selected brain regions were dissected, immediately frozen at $-80 \circ \mathrm{C}$, put in labeled plastic bags, and stored at $-80 \circ \mathrm{C}$ until use; the rest of the coronal sections were frozen and stored at $-80 \circ \mathrm{C}$ [24]. Paraffin sections were used as controls of positive staining in immunohistochemical techniques and Gallyas staining.

\section{Extraction of sarkosyl-insoluble fractions and western blotting}

Frozen samples of the hippocampus from one AD case (Braak and Braak stage $\mathrm{V}-\mathrm{VI} / \mathrm{C}$ ) and one case with no lesions were processed in parallel. The AD case did not show concomitant pathology and comorbidities; particularly, argyrophilic grains, thorn-shaped astrocytes, and coiled bodies were absent. most $\mathrm{m}$ Frozen samples of about $1 \mathrm{~g}$ were lysed in 10 volumes $(\mathrm{w} / \mathrm{v})$ with cold suspension buffer $(10 \mathrm{mM}$ Tris-HCl, pH 7.4, 0.8M NaCl, 1mM EGTA) supplemented with $10 \%$ sucrose, protease, and phosphatase inhibitors (Roche). The homogenates were first centrifuged at 20,000×g for $20 \mathrm{~min}$ (Ultracentrifuge Beckman with 70Ti rotor), and the supernatant (S1) was saved. The pellet was re-homogenized in five volumes of homogenization buffer and re-centrifuged at 20,000 $\mathrm{g}$ for $20 \mathrm{~min}$. The two supernatants (S1 + S2) were then mixed and incubated with $0.1 \% \mathrm{~N}$-lauroylsarkosynate (sarkosyl) for $1 \mathrm{~h}$ at room temperature while being shaken. Samples were then centrifuged at $100,000 \times \mathrm{g}$ for $1 \mathrm{~h}$. Sarkosyl-insoluble pellets $(\mathrm{P} 3)$ were re-suspended $(0.2 \mathrm{~mL} / \mathrm{g})$ in $50 \mathrm{mM}$ Tris- $\mathrm{HCl}(\mathrm{pH} 7.4)$. Protein concentrations were quantified with the bicinchoninic acid assay (BCA) assay (Pierce). Sarkosyl-insoluble fractions were processed for western blotting. Samples were mixed with loading sample buffer and heated at $95^{\circ} \mathrm{C}$ for 5 min. Sixty $\mu \mathrm{g}$ of protein was separated by electrophoresis in SDS-PAGE gels and transferred to nitrocellulose membranes (200mA per membrane, $90 \mathrm{~min}$ ). The membranes were blocked for $1 \mathrm{~h}$ at room temperature with $5 \%$ non-fat milk in TBS containing $0.2 \%$ Tween and were then incubated with the phospho-specific anti-tau Ser422 antibody (tau-P-Ser422) (diluted 1:1,000; Thermo Fisher Scientific). After washing with TBS-T, blots were incubated with the appropriate secondary antibody (anti-rabbit IgG conjugated with horseradish peroxidase diluted at 1:2,000, Agilent) for $45 \mathrm{~min}$ at room temperature. Immune complexes were revealed by incubating the membranes with chemiluminescence reagent (Amersham) $[22,23,26]$.

Sarkosyl-insoluble fractions of AD brain homogenates blotted with anti-tauSer422 were characterized by three bands of 68,64 and $60 \mathrm{kDa}$, a weak upper band of $73 \mathrm{kDa}$ and several lower bands of fragmented tau between 50 and $25 \mathrm{kDa}$. Strong lower bands stained with anti-tauSer422 indicated truncated tau at 
the C-terminal. This pattern is typical of 4Rtau+3Rtau tauopathies. Transmission electron microscopy analysis showed paired helical filaments. Thioflavin T amyloid quantification assay revealed positive curves of amyloid fibrils in sarkosyl-insoluble fractions from AD. Similar results have been detailed in our previous studies $[22,23,26]$. Sarkosyl-insoluble fractions of the control blotted with the same antibody were negative. The resultant sarkosyl-insoluble fractions from the $A D$ case and control case were used for inoculation.

\section{Animals}

The experiments were carried out in heterozygous transgenic mice expressing human P301S tau (line PS19: B6;C3-Tg(Prnp-MAPT*P301S)PS19Vle/J), homozygous transgenic mice knock-out for murine tau protein (mtWt), homozygous transgenic mice knock-out for murine tau protein and heterozygous expressing human forms of 3Rtau and 4Rtau proteins (hTau; B6.Cg-Mapttm1(EGFP)Klt $\operatorname{Tg}(\mathrm{MAPT}) 8 \mathrm{cPdav} / \mathrm{J}$ ), and wild-type (WT) littermates in a C57BL/ 6 background. The generation of mice expressing the human mutated form P301S tau and human forms of 3Rtau and 4Rtau protein is described by the supplier (Charles Rivers Laboratories). Transgenic mice were identified by genotyping genomic DNA isolated from tail clips using the polymerase chain reaction (PCR) conditions indicated by Jackson Laboratory (Bar Harbor, ME). Animals were maintained under standard animal housing conditions in a 12-hour dark-light cycle with free access to food and water. All animal procedures were carried out following the guidelines of the European Communities Council Directive 2010/63/EU and with the approval of the local ethical committee (C.E.E.A: Comitè Ètic d'Experimentació Animal; University of Barcelona, Spain; ref. 426/18). Animals were killed by decapitation and their brains were then rapidly removed and processed for study. The left cerebral hemisphere, brainstem, and cerebellum were dissected on ice, immediately frozen, and stored at $-80^{\circ} \mathrm{C}$ until use for biochemical studies. The right hemisphere, brainstem, and cerebellum were fixed in $4 \%$ paraformaldehyde, cut in coronal sections, and embedded in paraffin. De-waxed sections were stained with haematoxylin and eosin, and Gallyas staining, or processed for immunohistochemistry.

The immunohistochemical characterization of mice was carried out at 6 and 9/10 months, and the biochemical study in mice aged 9/10 months; four animals per group were assessed.

\section{Inoculation of sarkosyl-insoluble and from Alzheimer disease and control homogenates in the hippocampus of WT, P301S, hTau, and mtWt mice}

Mice were inoculated with the same AD sarkosyl-insoluble fraction to avoid differences related to the composition of the inoculum. One series of mice was inoculated at the age of 6 months and killed at the age of 9 months ( $n=6$ per group); the other series was inoculated at the age of 3 months and killed at the age of 9 months ( $n=6$ per group). Other groups of mice were inoculated with sarkosyl-insoluble fractions from control homogenates $(n=4)$. Not any group showed differences in survival times due the inoculum. One WT mouse was inoculated with vehicle $(50 \mathrm{mM}$ Tris- $\mathrm{HCl}, \mathrm{pH} 7.4)$ at the age of 3 months and killed at the age of 10 months. Mice were deeply anesthetized with an intra-peritoneal injection of ketamin/xylazine/buprenorphine cocktail and placed in a stereotaxic frame after assuring lack of 
reflexes. For the intra-hippocampal inoculation, we used a Hamilton syringe (volume $2.0 \mu \mathrm{L}$ ); the coordinates were $-1.9 \mathrm{~mm}$ interaural; $-1.4 \mathrm{~mm}$ relative to Bregma, and $-1.8 \mathrm{~mm}$ DV from the dural surface (Paxinos and Franklin, 2019). A volume of $1.5 \mu \mathrm{L}$ was injected at a rate of $0.05 \mu \mathrm{L} / \mathrm{min}$. The syringe was withdrawn slowly over a period of $10 \mathrm{~min}$ to avoid leakage of the inoculum. Following surgery, the animals were kept in a warm blanket and monitored until they recovered from the anesthesia. Carprofen analgesia was administered immediately after surgery and once a day during the next two consecutive days. Animals were housed individually with full access to food and water. Mice were by decapitation and the brains rapidly fixed with $4 \%$ paraformaldehyde in phosphate buffer, cut in coronal sections, and embedded in paraffin.

Another group of mice $(n=6)$ were inoculated with sarkosyl-insoluble fractions into the lateral ventricle. The coordinates were AP: $-0.6, \mathrm{ML}: \pm 1.2$ and DV: -2.2 for $15 \mathrm{~min}$, followed by a waiting time of $4 \mathrm{~min}$ and removal of the needle $15 \mathrm{~min}$ later. All the procedure was the same as used in the core group. The survival time was three months.

Lesions were assessed in consecutive sections consistent with coordinates -2.3 to -1.6 interaural, and -1.4 to -2.1 from Bregma.

\section{Immunohistochemistry}

De-waxed sections, 4 microns thick, were processed for immunohistochemistry. The sections were boiled in citrate buffer $\mathrm{pH}=6$ (20 $\mathrm{min}$ ) to retrieve tau antigenicity. Endogenous peroxidases were blocked by incubation in $10 \%$ methanol- $1 \% \mathrm{H}_{2} \mathrm{O}_{2}$ solution ( $15 \mathrm{~min}$ ) followed by $3 \%$ normal horse serum solution. The sections were incubated at $4^{\circ} \mathrm{C}$ overnight with one of the primary antibodies listed in Table 1. Following incubation with the primary antibody, the sections were incubated with EnVision + system peroxidase (Dako, DK) for $30 \mathrm{~min}$ at room temperature. The peroxidase reaction was visualized with diaminobenzidine and $\mathrm{H}_{2} \mathrm{O}_{2}$. Control of the immunostaining included omission of the primary antibody; no signal was obtained following incubation with only the secondary antibody.

Selected sections were processed with the method of in situ end-labeling of nuclear DNA fragmentation ApopTag Plus Peroxidase in situ Apoptosis Detection Kit (S 7101; Millipore) following the protocol of the supplier. Positive controls facilitated by the supplier and the cerebellum of X-irradiated newborn rats surviving $48 \mathrm{~h}$ were processed in parallel.

\section{Quantification of abnormal deposits revealed by immunohistochemistry}

Deposits in neurons and related axonal fibers and threads in the hippocampal complex were expressed semi-quantitatively considering the whole tissue per case in every case; the following signs indicate: +++: abundant; ++: moderate; +: scarce; +/-: not present in every case.

\section{Double-labeling immunofluorescence and confocal microscopy}


De-waxed sections, 4 microns thick, were stained with a saturated solution of Sudan black B (Merck) for 15 min to block autofluorescence of lipofuscin granules present in cell bodies, and then rinsed in $70 \%$ ethanol and washed in distilled water. The sections were boiled in citrate buffer to enhance antigenicity and blocked for $30 \mathrm{~min}$ at room temperature with $10 \%$ fetal bovine serum diluted in PBS. Then, the sections were incubated at $4^{\circ} \mathrm{C}$ overnight with combinations of primary antibodies against different proteins. The characteristics of the antibodies, dilutions, and suppliers are listed in Table 1. After washing, the sections were incubated with Alexa488 or Alexa546 (1:400, Molecular Probes) fluorescence secondary antibodies against the corresponding host species. Nuclei were stained with DRAQ5 ${ }^{\mathrm{TM}}$ (1:2,000, Biostatus). After washing, the sections were mounted in Immuno-Fluore mounting medium (ICN Biomedicals), sealed, and dried overnight. Sections were examined with a Leica TCS-SL confocal microscope. 
Table 1

List of antibodies used for immunohistochemistry and western blotting.

\begin{tabular}{|c|c|c|c|c|c|}
\hline Antibody & Supplier & Reference & Host & WB dil & IHQ dil \\
\hline 3Rtau & Upstate & $05-803$ & Ms & $1 / 1,000$ & $1 / 800$ \\
\hline 4Rtau & Millipore & $05-804$ & Ms & $1 / 1,000$ & $1 / 50$ \\
\hline AKT1-P Ser473 & Abcam & Ab18206 & $\mathrm{Rb}$ & - & 1:100 \\
\hline$\beta$-actin & Sigma & A5316 & Ms & $1 / 30,000$ & - \\
\hline CLK1 (75-100 aa) & LS Bio & LS-C382760 & Ms & - & $1 / 100$ \\
\hline CK1-ठ & Abcam & ab85320 & Ms & - & $1 / 500$ \\
\hline elF2a-P Ser51 & Thermo Scientific & MA5-15133 & $\mathrm{Rb}$ & - & $1 / 100$ \\
\hline GFAP & Dako & Z0334 & $\mathrm{Rb}$ & - & $1 / 400$ \\
\hline GSK-3 $\beta$-P Ser9 & Cell signaling & 9336 & $\mathrm{Rb}$ & - & $1 / 100$ \\
\hline Iba1 & Wako & 019-19741 & $\mathrm{Rb}$ & - & $1 / 1000$ \\
\hline IRE-P Ser274 & Abcam & $a b 48187$ & $\mathrm{Rb}$ & - & $1 / 100$ \\
\hline LAMP1 & Santa Cruz & Sc5570 & $\mathrm{Rb}$ & - & $1 / 10$ \\
\hline LC3 & Cell Signalling & 2775 & $\mathrm{Rb}$ & - & $1 / 100$ \\
\hline MAP2-P Thr1620/1623 & Cell Signaling & 4544 & $\mathrm{Rb}$ & - & $1 / 1,000$ \\
\hline NFL-P Ser473 & Millipore & MABN2431 & Ms & - & $1 / 100$ \\
\hline p38-P Thr180/Tyr182 & Cell Signaling & 9211 & $\mathrm{Rb}$ & - & $1 / 100$ \\
\hline PHF1, tau-P Ser396/Ser404 & Dr. Peter Davies & - & Ms & - & $1 / 500$ \\
\hline PKAa/ $\beta-P$ Tyr197 & Invitrogen & 44988 & $\mathrm{Rb}$ & & $1 / 100$ \\
\hline SAPK/JNK-P-Thr183/Thr185 & Cell Signaling & 9251 & $\mathrm{Rb}$ & - & $1 / 25$ \\
\hline SRC-P Tyr416 & Millipore & $05-677$ & Ms & - & $1 / 100$ \\
\hline SRPK1 & Abcam & ab90527 & $\mathrm{Rb}$ & - & $1 / 100$ \\
\hline
\end{tabular}

3Rtau: tau with three repeats; 4Rtau: tau with four repeats; AKT1: serine/threonine-protein kinase AKT1; $\beta$-actin; CK1- $\delta$ : casein kinase $\delta$; elF2a: eukaryotic initiation factor $2 a ; G S K ~ \beta$ : glycogen synthase kinase 3 $\beta$; IRE1: inositol-requiring enzyme 1 $\alpha$; LAMP1: lysosomal-associated membrane protein 3; LC3: microtubule-associated protein 1A/1B-light chain 3; MAP2: microtubule-associated protein 2; NFL: neurofilament light molecular weight; p38: p38-kinase; PHF1: anti-paired helical filament 1; PKAa/ $\beta$ : cAMP-dependent protein kinase A; SAPK/JNK: stress-activated protein kinase/Jun aminoterminal kinase; SRC: tyrosine-protein kinase SRC; Tau 100; Tau 5: monoclonal recognizing total tau protein; Tau AT8; Tau C3: tau truncated at aspartic acid 421; Tau MC1: conformation tau; Tau-N: nitrated tau; Tau-P phosphorylated tau. The phosphorylation or nitration of the protein at the specific site is indicated in every case. WB dil: western blotting dilution; IHQ dil: immunohistochemistry dilution. 


\begin{tabular}{|c|c|c|c|c|c|}
\hline Antibody & Supplier & Reference & Host & WB dil & IHQ dil \\
\hline SRPK2-P Ser497 & Affbiotech & 3632 & $\mathrm{Rb}$ & - & $1 / 100$ \\
\hline Tau 5 & Thermo Scientific & MA5-12808 & Ms & $1 / 500$ & - \\
\hline Tau AT8-P Ser202/Thr205 & Innogenetics & 90206 & Ms & - & $1 / 50$ \\
\hline Tau C-3 & Millipore & $36-017$ & Ms & - & $1 / 100$ \\
\hline Tau MC1 & Dr. Peter Davies & - & Ms & - & $1 / 20$ \\
\hline Tau-N Tyr29 & Millipore & MAB2244 & Ms & - & $1 / 200$ \\
\hline Tau-P Ser422 & Thermo Scientific & 44764 & $\mathrm{Rb}$ & - & $1 / 50$ \\
\hline Tau-P Thr181 & Thermo Scientific & PA1-14413 & $\mathrm{Rb}$ & $1 / 1,000$ & - \\
\hline Ubiquitin & Dako & Z0458 & Ms & - & $1 / 250$ \\
\hline \multicolumn{6}{|c|}{ 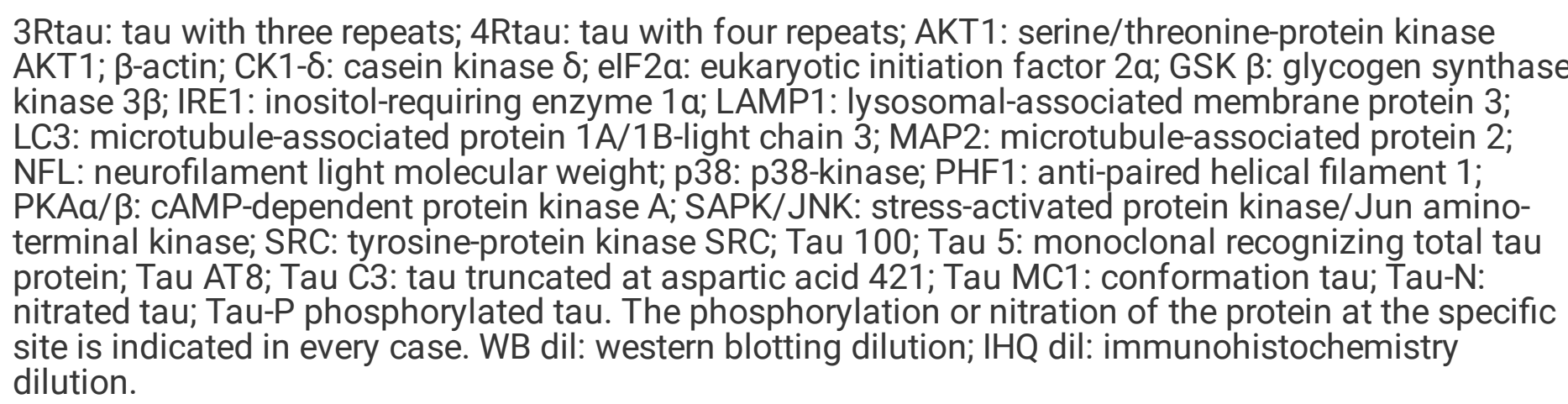 } \\
\hline
\end{tabular}

\section{Gel electrophoresis and western blotting}

Frozen samples of the whole mouse brain were homogenized in RIPA lysis buffer composed of $50 \mathrm{mM}$ Tris/HCl buffer, pH 7.4 containing $2 \mathrm{mM}$ EDTA, $0.2 \%$ Nonidet P-40, $1 \mathrm{mM}$ PMSF, protease and phosphatase inhibitor cocktail (Roche Molecular Systems, USA). The homogenates were centrifuged for $20 \mathrm{~min}$ at $12,000 \mathrm{rpm}$. Protein concentration was determined with the BCA method (Thermo Scientific). Equal amounts of protein $(12 \mu \mathrm{g})$ for each sample were loaded and separated by electrophoresis on $10 \%$ sodium dodecyl sulfate polyacrylamide gel electrophoresis (SDS-PAGE) gels and transferred onto nitrocellulose membranes (Amersham, Freiburg, GE). Non-specific bindings were blocked by incubation in $3 \%$ albumin in PBS containing $0.2 \%$ Tween for $1 \mathrm{~h}$ at room temperature. After washing, membranes were incubated overnight at $4^{\circ} \mathrm{C}$ with antibodies against different forms of tau protein (Table 1). Protein loading was monitored using an antibody against $\beta$-actin (42kDa, 1:30,000, Sigma). Membranes were incubated for $1 \mathrm{~h}$ with appropriate HRP-conjugated secondary antibodies (1:3,000, Dako); the immunoreaction was revealed with a chemiluminescence reagent ( $E C L, A m e r s h a m)$. Densitometric quantification was carried out with the ImageLab v4.5.2 software (BioRad), using $\beta$-actin for normalization. The brains of four P301S, four hTau, three WT, and three mtWT mice were processed for gel electrophoresis and western blotting. 


\section{Statistical analysis}

Results were analyzed statistically with SPSS 19.0 (SPSS Inc., USA) software and GraphPad PRISM (GraphPad Software, Inc.) software. Data were presented as mean \pm standard error of the mean (SEM). Data were compared with one-way analysis of variance (ANOVA) followed by post-hoc Tukey's multiple comparison test when necessary. Significance level was set at ${ }^{*} P<0.05 * * P<0.01, * \star * P<0.001 \mathrm{vs}$. Wt; $\# \mathrm{P}<0.05$, \#\#\# $\mathrm{P}<0.001$ vs. P301S; and $\$ \mathrm{P}<0.05$;

$$
P<0.01 \text { and }
$$

$\$ \mathrm{P}<0.001$ vs. mtWt.

\section{Results}

\section{P301S transgenic mice}

Transgenic mice at the age of 6 and 9/10 months showed phosphorylated tau deposition, as revealed with the AT8 antibody, in the cytoplasm of neurons and proximal dendrites in the entorhinal and piriform cortex, CA1 region of the hippocampus, dentate gyrus, somatosensory cortex, and cingulate cortex; the amygdala, hypothalamus, striatum, thalamus, septal nuclei, nuclei of the basal forebrain, and granule cells of the cerebellum were also immunostained with AT8 antibodies; neurons of the brainstem, particularly those in the pontine nuclei, tegmentum, and raphe nuclei also contained AT8-positive deposits (Supplementary Figure 1a-c). Neuropil threads in the grey matter, plexiform layers of the hippocampus, hilus of the dentate gyrus, and molecular layer of the cerebellum also contained AT8positive deposits. Antibodies PHF1, directed against tau-P Ser396/404, and phospho-tau Ser422 showed a similar distribution and localization of phosphorylated tau (Supplementary Figure 1d-i). The antibody tau C-3, directed against truncated tau at aspartic acid 421, stained only a few neurons in the CA1 region and dentate gyrus in mice aged 9/10 months (Supplementary Figure 1j). Tau deposits were homogeneous and diffuse and did not have the appearance of neurofibrillary tangles. Only a few neurons containing phospho-tau were positive with Gallyas staining. Astrocytes and oligodendrocytes were devoid of phosphorylated tau deposits. These findings were in agreement with our previous observations in P301S transgenic mice [28].

Immunohistochemistry with anti-4Rtau antibodies revealed a positive neuropil, but not increased 4Rtau deposits in any cell type. 3Rtau immunohistochemistry was negative, excepting for a few neurons in the polymorphic sub-layer of the dentate gyrus, piriform cortex, olfactory tubercle, reticular thalamic nuclei, and dorsomedial hypothalamic nucleus. Neurons also contained deposits of tau nitrated at Tyr29 (tau-N Tyr29) in the same regions and localizations as those revealed with AT8 antibodies (Supplementary Figure $1 \mathrm{k}, \mathrm{l}$ ). Neurons also contained phosphorylated microtubule associated protein, as recognized with the MAP2-P Thr1620-1623 antibody, with the same extension and localization as tau AT8 (Supplementary Figure 2a-c). In contrast, a few neurons, mainly localized in the hippocampus and 
cerebral cortex, exhibited phosphorylated light neurofilament (NFL-P Ser473) immunoreactivity (Supplementary Figure 2d).

A subpopulation of neurons was positive with anti-casein kinase $\delta$ antibodies (Supplementary Figure $2 \mathrm{e}, \mathrm{f})$. Subpopulations of neurons also showed cytoplasmic granules positive with antibodies against active p38 (p38-P Thr180/tyr182), phosphorylated tyrosine-protein kinase SRC (SRC-P Tyr416) (only in the entorhinal cortex), and phosphorylated stress activated protein kinase/Jun amino-terminal kinase (SAPK/JNK-P Thr183/185) (Supplementary Figure 2g-j). GSK-3ß-P Ser9 immunoreactivity was negative. Markers of reticulum stress elF2a-P Ser51 and IRE-P Ser274 were negative. Only a few neurons in the dentate gyrus and threads in the hilus showed ubiquitin deposits in mice aged 10 months (Supplementary Figure 2k). LC3-immunoredactive granules were not observed. A few cells with nuclear DNA fragmentation, as revealed with Apoptag, and a few cells positive with antibodies against truncated (active) caspase-3, were also identified in the dentate gyrus in P301S mice at the age of 9/10 months (data not shown) [58].

Immunostaining with AKT-P Ser473, PKAa/ $\beta-P$ Tyr197, SRPK1, and SRPK2-P Ser497 antibodies was negative excepting for SRPK1 immunoreactivity in neuronal and glial cell nuclei.

Double-labelling immunofluorescence and confocal microscopy to AT8 and active p38 showed colocalization of tau deposits and the active tau kinase in the cytoplasm (Supplementary Figure 3a,b). Similarly, neurons with AT8-immunoreactive deposits co-localized SAPK/JNK-P-positive granules in the cytoplasm (Supplementary Figure 3c,d). Double-labelling immunofluorescence and confocal microscopy to casein kinase and LAMP1 disclosed a unique image of co-localization. We did not find evidence of LAMP1-immunoreactive vacuoles.

The method of in situ end-labelling of DNA fragmentation showed a few positive cells with morphological features of apoptosis in the inner region of the dentate gyrus and hilus in animals aged 9/10 months, in agreement with previous observations [58].

\section{Human tau transgenic mice (hTau) and mutant wild-type KO for murine tau (mtWt) transgenic mice}

As expected, the brain of mtWt mice was not stained with anti-tau antibodies. In contrast, hTau transgenic mice showed MC1 and PHF1 immunoreactivity in the cytoplasm of neurons in the entorhinal cortex, hippocampus, dentate gyrus, and cerebral cortex. Positive neurons were rarely seen in the thalamus, but they were absent in the striatum and cerebellum. MC1-immunoreactive deposits were often crescent in shape and located in the cytoplasm of a subpopulation of neurons in mice aged 6 and 9/10 months. PHF1-immunoreactive neurons were also distributed at random in the same regions but they decorated the whole cytoplasm. In contrast with P301S transgenic mice, the cytoplasm of neurons in hTau mice was not stained with antibodies AT8 and tau-P Ser422. Gallyas staining was negative. Main characteristics of mtWT and hTau compared with WT and P301S mice are shown in Figure 1. Images of the $\mathrm{CA} 1$ region of the hippocampus and dentate gyrus are useful for comparison with the corresponding regions in inoculated mice (see later). 
In contrast with P301S transgenic mice, neurons in hTau mice were not stained with antibodies tau C-3, nitrated tau at TyrSer29, MAP2-P Thr1620/1623, NFL-P Ser473, casein kinase $\delta$, p38-P Thr180/tyr182, SAPK/JNK-P Thr183/Thr185, and SRC-P Tyr416. Ubiquitin deposits were absent. We did not detect abnormal structures with anti-elF2a-P Ser51, IRE-P Ser274 GSK-3 $\beta$-P Ser9, LAMP1, and LC3 antibodies (data not shown). AKT-P Ser473, PKAa/ $\beta-P$ Tyr197, SRPK1, and SRPK2-P Ser497 antibodies did not show abnormal deposits.

No cells showed nuclear DNA fragmentation, as revealed with Apoptag, in any region or at any age in hTau and mtWt transgenic mice (data not shown).

\section{Differences in neuronal deposits in WT, P301S, hTau, and mutant wild-type KO for murine tau (mtWt) transgenic mice}

WT mice showed 4Rtau immunoreactivity in the neuropil but 3Rtau immunoreactivity was negative as expected for adult WT mice. The antibodies PHF1 showed diffuse immunoreactivity in the neuropil without deposits in the cytoplasm of neurons and dendrites, whereas MC1 immunoreactivity was negative. The antibody Tau 100 slightly decorated the nuclei of neurons. The antibody AT8 stained the nuclei of neurons in WT mice but not the cellular processes.

P301S mice showed marked 4Rtau immunoreactivity in the neuropil without the appearance of local deposits in any particular neuronal subpopulation. Immunostaining with anti-3Rtau antibodies was negative. The antibody PHF1 showed strong immnoreactivity in the neuronal bodies and dendrites; the MC1 antibody disclosed selective neuronal deposits in a smaller percentage of neurons at this age. Immunoreactivity to Tau 100 occurred in the cytoplasm of a small number of neurons, in addition to weak nuclear staining. In contrast, as detailed above, strong AT8 immunoreactivity occurred in the cell body and dendrites of cortical neurons in P301S mice, in addition to nuclear staining.

4Rtau immunoreactivity in hTau mice was weak in contrast with the strong 3Rtau immunoreactivity in neurons and neuropil. PHF1 and MC1 antibodies weakly decorated the cytoplasm of a subpopulation of cortical neurons in hTau.

Finally, neurons and neuropil in mtWT mice were not stained with anti-3Rtau, 4Rtau, PHF1, MC1, and Tau 100 antibodies. AT8 nuclear staining in WT, mtWT and hTau was non-specific and was the result of an unknown cross-reaction.

Gallyas staining was negative in WT, mtWT, and hTau, whereas a few neurons were positive in P301S transgenic mice.

Differences between P301S and hTau are summarized in Table 2.

Western blotting of total brain homogenates in WT, P301S, hTau, and mutant wild-type KO for murine tau $(\mathrm{mtWt})$ 
Total brain homogenates were used to identify tau profiles in the four groups of mice at the age of 9/10 months. The antibody Tau 5 that identifies total tau showed strong bands in P301S and hTau animals when compared with WT mice. As expected, mtWt mice were negative. 4Rtau was present in WT mice, but it was strongly increased in P301S and to a lesser degree in hTau mice. In contrast, 3Rtau was absent in WT and P301S transgenic mice, but it was abundant in hTau mice. Therefore, 3Rtau was predominant when compared with 4Rtau in hTau transgenic animals. Phosphorylated tau at Thr181 was present in P301S and hTau and almost absent in WT mice. Curiously, PHF1 antibodies recognized a unique band of about $64 \mathrm{kDa}$ in WT mice. We do not know the origin of this band, but it matches with the diffuse PHF1 immunostaining of the neuropil in WT mice as revealed by immunohistochemistry. The pattern of PHF1 in WT mice largely differed from the PHF1 tau phospho-tau profile in P301S and hTau transgenic mice. Finally, MC1 antibodies recognized bands in P301S and hTau transgenic mice but not in WT mice. As expected, mtWt transgenic mice were negative with 3Rtau, 4Rtau, tau-P Thr181, PHF1, and MC1 antibodies (Figure 5).

The tau band pattern also differed between P301S and hTau transgenic mice. Two bands corresponding to 4Rtau were characteristic of P301S mice as revealed with 4Rtau and PHF1 antibodies. Three-four main bands corresponding to 3Rtau plus 4Rtau were revealed with the antibody PHF1 in hTau. A different profile was obtained with the antibody MC1; a doublet of high molecular weight was identified in P301S mice, but four bands were obtained in hTau mice, thus indicating different tau conformational changes in P301S and hTau mice (Figure 2).

The diagram in Figure 2 shows the results of the densitometric analysis of tau bands in the four groups of animals. Although the number of animals was relatively small ( $n=4$ per group), the patterns were sufficiently different among groups to permit a confident evaluation.

\section{Inoculation of mice with sarkosyl-insoluble of AD: characteristics of tau seeding in WT, P301S, and hTau genotypes. Lack of tau seeding in mtWT mice}

Mice inoculated with vehicle or with tau homogenates into the lateral ventricle did not show tau deposits at seven and three months, respectively, after inoculation.

Inoculated mice showed local astrocytic gliosis and variable number of phagocytes filled with haemosiderin and clear vacuoles along the course of the needle and the site of the injection independently of the genotype. Areas distant from the injection site, including the contralateral dentate gyrus, did not show increased numbers of astrocytes and microglia. Tau deposits were not detected along the trajectory of the needle in animals inoculated with sarkosyl-insoluble fractions from $A D$ and control homogenates. These changes were interpreted the result of non-specific traumatic injury of the intracerebral injection.

Lesions were assessed in consecutive sections consistent with coordinates -2.3 to -1.6 interaural, and -1.4 to -2.1 from Bregma. 
WT mice unilaterally inoculated with AD sarkosyl-insoluble fractions in the hippocampus/dentate gyrus at six months and killed at nine months showed abnormal tau deposition, as revealed with the antibody AT8, in neurons of the dentate gyrus, cerebral cortex near the site of inoculation, CA1 region of the hippocampus, and threads and glial cells in the fimbria and ipsilateral corpus callosum (Figure 3a-d). Abnormal deposits were also strongly immunostained with anti-MAP2-P Thr1620/1623 antibodies (Figure 3e-h).

P301S mice unilaterally inoculated in the hippocampus/dentate gyrus with AD sarkosyl-insoluble fractions at six months and killed at nine months showed abnormal tau deposition localized in neurons, threads, and glial cells with a pattern similar to that seen in WT mice. The distribution was also similar involving the dentate gyrus, CA1 region, cerebral cortex near the side of inoculation, corpus callosum, and fimbria. The main difference with WT mice was the abundance of deposits, higher in P301S when compared with WT mice. This was particularly remarkable regarding tau deposits in glial cells (Figure 3in). In the white matter, large numbers of glial cells and threads were found in the ipsilateral corpus callous, fewer numbers in the middle corpus callosum, and very few or no cells and threads in the proximal part of the contralateral corpus callosum (Figure 3j-I). Similarly, large numbers of glial cells and threads were immunostained in the ipsilateral fimbria, but lesser numbers in the contralateral fimbria (Figure 3m,n). MAP2-P Thr1620/1623 antibodies also decorated abnormal deposits in the same regions (Figure 3o-q).

mtWt mice did not show tau seeding and spreading following intrahippocampal inoculation with sarkosyl-insoluble fractions of $A D$ homogenates at three months of survival. The only presence of pigmented debris occurred in a few macrophages at the site of injection (data not shown).

hTau mice inoculated in the hippocampus/dentate gyrus with AD sarkosyl-insoluble fractions at six months and killed at nine months showed abnormal tau deposition in the cerebral cortex near the inoculation site, corpus callosum, CA1 region of the hippocampus, dentate gyrus, hilus, and fimbria (Figure 4a-e). The deposits were localized in neurons, threads, and granules. Deposition in glial cells was rare when compared with the number of glial deposits in inoculated WT and P301S transgenic mice. Deposits contained 3Rtau, but the abnormal deposits were mainly made up of 4Rtau (Figure 4f-i). The morphology of neuronal deposits also differed in shape as they were larger and flame-shaped in hTau mice. Differences were also obvious as the spreading in the corpus callosum and the involvement of oligodendrocytres were more restricted in hTau when compared with inoculated WT and P301S mice.

Double-labelling immunofluorescence and confocal microscopy to p38-P and AT8, and SAPK/JNK-P and AT8 in inoculated WT, P301S and hTau showed the co-localization of these active tau kinases and hyperphosphorylated tau deposition (Figure 5).

The alterations were similar in mice inoculated at the age of three months and killed six months later (survival time six months). Neurons in inoculated animals were Gallyas-negative despite the genotype (with the exception of a few basal Gallyas-positive neurons in P301S mice). In contrast, oligodendrocytes with abnormal deposits were Gallyas-positive in WT and P301S. A few Gallyas-positive oligodendrocytes 
were only seldomly observed in inoculated hTau, probably due to the reduced involvement of oligodendrocytes in hTau when compared with WT and P301S mice (Supplementary Figure 4). Neuronal and glial deposits in inoculated mice were devoid of reticulum stress markers elF2a-P Ser51, IRE-P Ser274, and markers of autophagy LC3 and LAMP1. No apoptotic cells, as revealed with the method of in situ end-labeling of nuclear DNA fragmentation, were seen in any cases.

Tau deposits were not detected in animals inoculated with homogenates from control brains. No tau deposits were seen in mice inoculated with vehicle alone.

\section{Differences of tau seeding and spreading among genotypes}

A summary of the main characteristics of deposits in P301S, hTau, and inoculated WT, P301S, and hTau in mice inoculated with identical $A D$ inoculums is shown in Table 2. 
Table 2

Characteristics of tau deposits in P301S and hTau mice, and WT, P301S and hTau mice unilaterally inoculated into the hippocampus with the same AD-tau enriched fractions.

\begin{tabular}{|c|c|c|c|c|c|}
\hline Antibody & P301S & hTau & Inocul WT & Inocul P301S & Inocul hTau \\
\hline 3Rtau & $-*$ & diffuse & + & + & $++(\mathrm{nft})$ \\
\hline 4Rtau & diffuse & - & + & + & $+++(\mathrm{nft})$ \\
\hline AKT-P Ser473 & - & - & - & - & $++(\mathrm{gr})$ \\
\hline CLK1 & - & - & - & - & $++(\mathrm{gr})$ \\
\hline CK1- $\delta$ & + (rare) & - & - & $+($ rare $)$ & $++(\mathrm{gr})$ \\
\hline elF2a-P Ser51 & - & - & - & - & - \\
\hline GSK-3ß-P Ser9 & - & - & - & - & $++(\mathrm{gr})$ \\
\hline IRE-P Ser274 & - & - & - & - & - \\
\hline LAMP1 & - & - & - & - & - \\
\hline LC3 & - & - & - & - & - \\
\hline MAP2-P Thr1620/1623 & $++(p t g)$ & - & $+(p t g)$ & $++(p t g)$ & $+++(\mathrm{nft})$ \\
\hline NFL-P Ser473 & $+(\mathrm{ptg})$ & - & - & - & - \\
\hline p38-P Thr180/Tyr182 & $++(g r)$ & - & $++(g r)$ & $++(g r)$ & $++(g r)$ \\
\hline PHF1, tau-P Ser396/Ser404 & $+(\mathrm{pt})$ & $+/-(\mathrm{gl})$ & - & $+(\mathrm{pt})$ & $++(\mathrm{nft})$ \\
\hline PKAa/ß-P Tyr197 & - & - & - & - & $++(\mathrm{gr})$ \\
\hline SAPK/JNK-P-Thr183/Thr185 & $++(\mathrm{gr})$ & - & $++(g r)$ & $++(\mathrm{gr})$ & $++(\mathrm{gr})$ \\
\hline SRC-P Tyr416 & $+(\mathrm{EC})$ & - & - & $+(E C)$ & - \\
\hline SRPK1 & - & - & - & - & - \\
\hline SRPK2-P Ser497 & - & - & - & - & - \\
\hline Tau AT8-P Ser202/Thr205 & $+++(p t g)$ & - & $+++(p t g)$ & $+++(p t g)$ & $+++(\mathrm{nft})$ \\
\hline Tau C-3 & $+($ rare $)$ & - & - & $+($ rare $)$ & - \\
\hline
\end{tabular}

Deposits in inoculated mice occur in neurons and threads in the three genotypes, and oligodendroglia (coiled bodies) in WT and P301S. The table refers to deposits in neurons and related axonal fibers and threads in the hippocampal complex. elF2a-P Ser51-immunoreactive is positive only in a few neuronal nuclei in inoculated and non-inoculated mice. SRPK1 and SRPK2-P Ser497 show granular immunoreactivity in the cytoplasm in non-inoculated and inoculated mice. nft: neurofibrillary tangles, ptg: pre-tangles; gr: granular deposits; gl: globular deposits. EC: entorhinal cortex. Age of noninoculated and inoculated mice at the time of examination: 9/10 months. The results are expressed semi-quantitatively considering the whole tissue per case in every case; the following signs indicate: +++: abundant; ++: moderate; +: scarce; +/-: not present in every case. 


\begin{tabular}{|c|c|c|c|c|c|}
\hline Antibody & P301S & hTau & Inocul WT & Inocul P301S & Inocul hTau \\
\hline Tau MC1 & $++(p t g)$ & $+/-(g l)$ & $+(p t g)$ & $++(p t g)$ & $+++(\mathrm{nft})$ \\
\hline Tau-N Tyr29 & $+(\mathrm{ptg})$ & - & - & - & $++(n f t)$ \\
\hline Tau-P Ser422 & $++(p t g)$ & - & $++(p t g)$ & $++(p t g)$ & $+++\mathrm{nft})$ \\
\hline Ubiquitin & +/- (hilus) & - & - & +/- (hilus) & - \\
\hline \multicolumn{6}{|c|}{$\begin{array}{l}\text { Deposits in inoculated mice occur in neurons and threads in the three genotypes, and oligodendroglia } \\
\text { (coiled bodies) in WT and P301S. The table refers to deposits in neurons and related axonal fibers } \\
\text { and threads in the hippocampal complex. elF2a-P Ser51-immunoreactive is positive only in a few } \\
\text { neuronal nuclei in inoculated and non-inoculated mice. SRPK1 and SRPK2-P Ser497 show granular } \\
\text { immunoreactivity in the cytoplasm in non-inoculated and inoculated mice. nft: neurofibrillary tangles, } \\
\text { ptg: pre-tangles; gr: granular deposits; gl: globular deposits. EC: entorhinal cortex. Age of non- } \\
\text { inoculated and inoculated mice at the time of examination: } 9 / 10 \text { months. The results are expressed } \\
\text { semi-quaantitatively considering the whole tissue per case in every case; the following signs indicate: } \\
+++: \text { abundant; ++: moderate; +: scarce; +/-: not present in every case. }\end{array}$} \\
\hline
\end{tabular}

Results are expressed semi-quantitatively, as densitometric studies of all antibodies in every case are unrealistic due to individual variations. Differences referred to regional vulnerability, morphology of the deposits, predominance of 3Rtau or 4Rtau deposits, immunochemical composition of the deposits including active kinases, and distinct vulnerability of oligodendrocytes were the most remarkable.

The involvement of the CA1 region was higher in hTau mice when compared with P301 and, particularly, with WT mice. In contrast, the contralateral corpus callosum and tau deposits in the fimbria were more marked in WT and P301S mice. Finally, tau deposits in the stratum radiatum and stratum oriens were more abundant in hTau in mice than in WT and P301S mice at the same survival time.

Neuronal tau deposits were diffuse cytoplasmic in inoculated P301S, but globular or flame-shaped in CA1 neurons in hTau. Different morphologies were consistent with pre-tangles in P301S and reminiscent of neurofibrillary tangles in hTau mice (Figures 6a,d, and Figure 7a,d).

4Rtau immunoreactivity in the CA1 region was similar in non-inoculated and inoculated P301S mice and $3 R$ tau was increased in a few neurons of the CA1 region. Curiously, 4Rtau was markedly augmented in neurons of the dentate gyrus and neuropil in the ipsilateral and contralateral hemispheres, and in mossy fibers in P301S mice (Figure 6b,c,e,f);

Regarding hTau mice, weak 4Rtau neuronal deposits occurred in the CA1 region and dentate gyrus, whereas neuronal deposits were strongly immunoreactive with anti-3Rtau antibodies in the CA1 region and dentate gyrus (Figure $7 b, c, e, f)$.

The composition of abnormal deposits identified with anti-tau-P Ser422, PHF1, MC1, tau N-Tyr29, and MAP2-P Thr1620/1623 antibodies were different in P301S and hTau. Moderate or weak tau-P Ser422, PHF1, and MAP2-P immunoreactivity in P301S mice was in contrast with the strong immunoreactivity in 
inoculated hTau. MC1 was negative and tau-N Tyr29 negative or sparsely positive in P301S, whereas MC1 and tau-N Tyr29 immunoreactivity was strong in inoculated hTau mice (Figure 6g-k and Figure 7g-k).

Expression of active (phosphorylated) kinases p38-P and SAP/JNK-P was observed in recruited phosphotau deposits in inoculated WT, P301S, and hTau mice, as detailed above. However, other kinases were

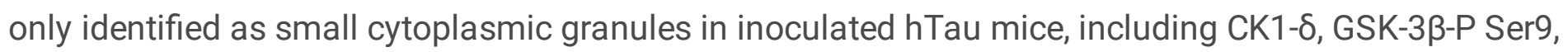
AKT-P Ser473, and PKAa/ $\beta-P$ Tyr197 in neurons with tau deposits. The expression of these kinases was absent in inoculated P301S mice (Figure 6I-o, Figure 7l-o, Figure 8a), and inoculated WT mice (not shown).

Finally, phosphorylated tau deposits in oligodendrocytes were abundant in inoculated WT and P301S mice, but scanty in hTau mice.

A final comment on the expression of SR protein kinases SRPK1 and SRPK2-P Ser497 is in order. Both kinases were expressed in the cytoplasm of neurons in non-inoculated and inoculated mice despite the genotype. Double-labeling immunofluorescence and confocal microscopy to AT8 and SRPK2-P Ser497 further revealed no differences between neurons without and with abnormal tau deposits in inoculated P301S and hTau (Figure 8b,c). Curiously, reactive astrocytes at the site of injection showed strong SRPK1 immunoreactivity in the cytoplasm and cell processes (data not shown). As a differential point between inoculated mice, punctate cytoplasmic CLK1 immunoreactivity was only observed in neurons of the CA1 region in inoculated hTau mice (Figure 8d).

\section{Discussion}

Several studies have demonstrated the capacity for seeding and spreading of tau-enriched fractions of brain homogenates from $A D$ and other human and mouse tauopathies following intracerebral inoculation into transgenic mice bearing human tau or mutant human tau $[8,9,11,12,16]$ and into WT mice $[9,13-$ $16,22-26]$.

In addition to neurons, deposits occur in glial cells, and the morphology of glial inclusions appears to mimic the glial aggregates of the corresponding human tauopathies in inoculated transgenic mice expressing human tau or mutant human tau [10-16]. However, our previous studies show differences between the phenotype of the original tauopathy and the resultant tauopathy following intracerebral inoculation of tau-enriched homogenates into WT mice [2-25]. In our experiments using WT mice, tau deposits in neurons resemble pre-tangles rather than tangles; oligodendrocytes contain tau deposits despite the tauopathy; and tau deposits are almost absent in astrocytes excepting first stages following tau ARTAG. Due to this uniform pattern following inoculation in WT mice, we speculated that host tau, in addition to inoculated tau, plays a role in the capacity for and pattern of seeding and spreading of tau [26].

\section{Four tau genotypes used in the study}


To assess this hypothesis, here we used four genotypes of mice carrying different forms of tau: adult WT mice expressing 4R murine tau, P301S transgenic mice expressing the human tau mutation P301S, hTau expressing mainly human 3Rtau and lesser amounts of $4 \mathrm{Rtau}$ in a $\mathrm{KO}$ murine tau background, and mtWT mice that do not express murine and human tau.

P301S transgenic mice at the age of 6 and 9/10 months show hyper-phosphorylated tau deposits in neurons with the appearance of pre-tangles in the cerebral cortex, amygdala, hippocampus, striatum, and thalamus; and neuropil threads in the cerebral cortex, and plexiform layers of the hippocampus. Tau deposits are positive with phospho-specific anti-tau antibodies AT8 (Ser202/Thr205), PHF1 (Ser396/404), Tau-P Ser422, Tau 100 (Tau-P Thr212/Ser214), and antibodies against epitopes within amino acids 312322 (conformational antibody MC1). Truncated tau at the aspartic acid 421 (Tau C-3) is also expressed in a subset of neurons at the age of 9/10 months [28]. Affected neurons in P301S transgenic mice also contain nitrated tau (Tau-N Tyr29). Although there is a positive 4Rtau background, neuronal tau deposits do not show 4Rtau immunoreactive enhancement.

Tau phosphorylation is accompanied by activation of p38, SAPK/JNK, and SRC kinases, as identified with the antibodies p38-P Thr180/182, SAPK/JNK-P Thr183/Thr185, and SRC-P Tyr416, respectively, but not by phosphorylation of GSK-3 $\beta$ Ser9. Affected neurons also show rare granular casein kinase $\delta$ immunoreactivity. However, markers of granulovacuolar degeneration linked to endoplasmic reticulum stress and autophagy (elF2a-P Ser51, IRE1-P Ser 274, LC3 and LAMP1) are negative, in agreement with recent observations [29]. In addition to phospho-tau, neuronal deposits are strongly immunoreactive with the MAP2-P Thr1620/1623 antibody. Astrocytes and oligodendrocytes barely contain abnormal tau deposits up to the age of $9 / 10$ months in our series.

hTau transgenic mice express high levels of human 3Rtau and low levels of 4Rtau in western blots of brain homogenates. Phosphorylated tau deposition occurs in subpopulations of neurons in the cerebral cortex and hippocampus. Abnormal deposits are identified with the antibodies PHF1 and MC1, but tau deposits are not stained with AT8, tau-P Ser422, and Tau C-3 antibodies. This phenotype is similar to that described previously in hTau transgenic mice [30]. Moreover, tau deposits in hTau do not contain Tau-N Tyr29. This contrasts with the presence of Tau-NTyr29 in NFT, dystrophic neurites, and neuropil threads in AD and NFTs in CBD and PSP [31] and P301S transgenic mice. There is no apparent activation of tau kinases p38, SAPK/JNK, GSK-3 $\beta$ Ser9, and SRC kinases co-localizing with abnormal tau deposits in hTau mice. Phosphorylated MAP2 is not deposited in neurons. Casein kinase $\delta$ immunoreactivity and markers of granulovacuolar degeneration linked to endoplasmic reticulum stress and autophagy are negative in hTau. Differences and similarities between P301S and hTau are shown in Table 2.

As expected, WT mice do not have tauopathy, and mtWT mice do not express any type of tau using the same battery of anti-tau antibodies.

\section{Inoculation of mice: variability of tau seeding and spreading depending on the host tau genotype.}


We inoculated tau fibrils from the same $A D$ case to randomize donor tau, and injected the same amount of the inocula unilaterally into the same region of the hippocampus in every case. Inoculations in the lateral ventricle, carried out to assess the capacity for seeding of tau fibrils in the CSF, resulted negative. This observation is in line with the failure to detect seeding of the CSF from AD using ultrasensitive tau biosensors [32].

Tau seeding and spreading does not occur in the absence of host tau, as no deposits develop in mtWt mice. This is particularly important as it indicates that host tau is essential for tau spreading in inoculated mice. Moreover, the latter experiment shows that the pathology of tau progression in inoculated mice is not due to the passive extension of the inocula.

The distribution of tau spreading following inoculation does not grossly differ in WT, P301S, and hTau mice inoculated at six months with a survival of three months, and in mice inoculated at three months and killed at the age of 9/10 months. Deposits of hyper-phosphorylated tau occur in neurons of the ipsilateral hippocampus, dentate gyrus, and cerebral cortex near the site of inoculation, and in threads in the corpus callosum and ipsilateral callosal radiation. The present results confirm pioneering observations demonstrating that the pattern of neuronal tau spread is largely determined by connectivity [8].

However, differences in the regional vulnerability to tau seeding and spreading depend on the genotype. The involvement of the CA1 region was higher in hTau mice when compared with P301 and, particularly, with WT mice. Tau deposits in the stratum radiatum and stratum oriens were more abundant in hTau in mice than in WT and P301S mice at the same survival time. In contrast, the contralateral corpus callosum and tau deposits in the fimbria were more marked in WT and P301S mice.

In addition to neurons, oligodendroglial cells in the fimbria, corpus callosum, and white matter contain hyper-phosphorylated tau, a feature not seen in AD. It is worth noting that oligodendrocytes in normal conditions produce and express tau protein [33,34]. Abnormal tau deposition in oligodendrocytes occurs constantly in our paradigms following inoculation of tau from different tauopathies including AD, PART, AGD, PSP, ARTAG, and FTLD-P301L in WT mice [22-26]. Oligodendroglial participation is also observed following tau inoculation from CBD homogenates in humanized tau mice [35]. These observations show that oligodendrocytes are principal participants in tau spreading following tau inoculation in mice expressing murine or human mutant tau. It is not clear whether tau spreading in oligodendrocytes occurs by continuity rather than connectivity. Differential vulnerability of oligodendrocytes is also dependent on the host tau genotype. Phospho-tau deposits in oligodendoglial cells are abundant in WT and P301S inoculated mice with AD-tau, but scanty if at all present in hTau transgenic mice. Since the involvement of oligodendrocytes differs in inoculated WT and P301S mice when compared with hTau mice, it may be inferred that oligodendrocyte vulnerability to identical tau inocula is also partially dependent on the host tau.

No evidence of unfolded protein response, autophagy, and apoptosis following tau inoculation 
Abnormal deposits in neurons and glial cells are not stained with anti-elF2a-P Ser51, IRE-P Ser274, LC3, and LAMP1 antibodies, suggesting that they do not present the biochemical markers of the unfolded protein response, and they aren't either consistent with granulovacuolar degeneration [36, 37]. These observations are in contrast with previous studies stressing the activation of the unfolded protein response in pre-tangle neurons and the induction of granulovacuolar degeneration bodies by intracellular tau pathology $[38,39]$. The discrepancies may originate in differences between human $A D$ and $A D$-tau inoculation in mouse models, and in the methods employed, more precisely between tau seeding in cultured neurons versus tau seeding and spreading in vivo.

In the same line, although tau hyper-phosphorylation may produce oxidative stress and neuronal death by apoptosis [40], apoptosis is only seen in the hilus and dentate gyrus in P301S mice aged 9/10 months, but not in hTau mice. Additional apoptosis is not observed in inoculated WT, P301S, and hTau mice at three and six months after tau inoculation.

\section{Post-translational modifications of recruited tau in inoculated mice depend on the tau genotype of the host}

Tau inoculation triggers an active process of post-translational modifications in vulnerable cells which is partially dependent on the host genotype. This is manifested by the appearance of new sites of tau phosphorylation and nitration in affected neurons in inoculated mice compared with the corresponding sites under baseline conditions in the different genotypes. Tau deposits in inoculated WT, P301S, and hTau are stained with antibodies AT8 and Tau-P Ser422 whereas no such deposit types occur in WT and hTau transgenic mice under normal conditions. This observation suggests that $A D$ tau has the capacity to induce post-translational modifications in the host tau which are reminiscent of $A D$ tau. Activation of p38 and SAPK-JNK co-localizing tau deposits in inoculated WT, P301S, and hTau mice further points to the active phosphorylation of retrieved tau from the host following tau inoculation, as already reported in WT mice inoculated with sarkosyl-insoluble brain fractions from various human tauopathies [22-26]. However, the characteristics of tau deposits in inoculated mice differ among genotypes. The morphology and composition of neuronal tau deposits are similar in inoculated WT and P301S mice, and they are reminiscent of pre-tangles. In contrast, neuronal tau aggregates in inoculated hTau mice resemble neurofibrillary tangles. This striking difference is manifested using a wide variety of specific antibodies including 3Rtau, 4Rtau, AT8, PHF1, tau-PSer422, tau MC1, and MAP2-P.

Moreover, abnormal tau protein aggregates in inoculated hTau mice, but not in WT and P301S mice, contain tau-N Tyr29, and co-localize with cytoplasmic granules immunoreactive with CK1- $\delta, ~ C L K 1$, GSK3ß-P Ser9, and PKAa/ $\beta-P$ Tyr197 antibodies.

\section{Modulation of tau transcription in the host following identical inoculum is host genotypedependent}

Tau deposits in inoculated mice with AD fibrils are composed of 4Rtau and 3Rtau. Murine 4Rtau and human mutant 4Rtau predominate in WT and P301S mice, respectively; and human 3Rtau predominates in hTau transgenic mice. Yet, the amount of 3Rtau and 4Rtau isoforms produced in inoculated mice 
varies from one genotype to another. However, deposits of 3Rtau prevail in neuronal deposits in inoculated WT and P301S transgenic mice, whereas deposits of 4Rtau prevail in hTau transgenic mice. Not only is 3Rtau expressed de novo in mice with predominance of 4Rtau, but the composition of de novo tau deposits in the inoculated mice is the reverse of that expressed in the corresponding non-inoculated mice.

Curiously, modifications in the expression of tau isoforms have been observed in oligodendrocytes following middle cerebral occlusion in rats and mice. Some peri-infarct oligodendrocytes shift from 4Rtau expression to 3Rtau isoform immunoreactivity thus suggesting exon 10 splicing plasticity under determinate conditions [41].

Several factors may contribute to differences between the different genotypes. One of them is species differences of tau [42].

A shift from foetal to adult tau isoform expression occurs in mice and humans [43]. Exon 10 splicing rests on the activity of various SR proteins and diverse RNA-interacting and RNA/DNA-binding proteins [44]. SR protein kinase (SRPK) and cdc-like kinase (CLK/Sty) phosphorylate SR proteins and control their functions [45-47]. Other kinases, such as cAMP-dependent protein kinase (PKA), serine/threonine-

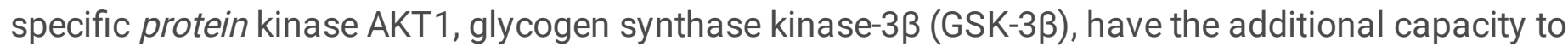
phosphorylate selected SR proteins [48-51].

The present observations in hTau mice inoculated with AD-tau show the activation by phosphorylation at specific sites of PKA, AKT1, GSK-3 $\beta$, and CLK1 in neurons, mainly in the CA1 region, containing higher expression of 4 Rtau than $3 R$ tau. Although these kinases might be involved in altered tau transcription in inoculated hTau mice, this scenario does not apply to inoculated WT and P301S mice.

Several tau fractions in $A D$ have differential prion-like activities [4]. Following this line of thinking, the diversity of tau strains may contribute to clinical diversity in $\operatorname{AD}[52,53]$. As a working hypothesis, it may be postulated that variable composition of $3 \mathrm{Rtau}$ and $4 \mathrm{Rtau}$ isoforms is expressed in different brain regions and in different neurons in a particular individual. Baseline differences in the composition of tau would modulate the vulnerability of specific neuronal and glial populations to the presence of pathological tau strains. The present findings may have implications in human tauopathies. Individual neurons and glial cells may have differences in tau composition. Therefore, tau variability, in addition to other factors, would contribute to individual regional and cell vulnerability not only in the context of different tauopathies but also in the phenotypic variability between individuals affected by the same tauopathy.

\section{Conclusions}

The present study reveals that a) host tau is mandatory for tau seeding and spreading following tau inoculation; b) tau seeding and spreading is characterized by major genotype-dependent biochemical changes linked to post-translational tau modifications including tau phosphorylation and tau nitration at 
different sites, c) tau seeding and spreading is accompanied by genotype-dependent activation of various kinases thus pointing to a complex molecular response in the receptive host cells; tau hyperphosphorylation of host tau is an active process implicating specific kinases; d) tau seeding and spreading is accompanied by modifications in tau splicing with variable expression of new 3Rtau and 4Rtau isoforms, thus indicating that inoculated tau seeds have the capacity to model exon 10 splicing of the host mapt with a genotype-dependent pattern; e) selective regional and cellular vulnerabilities, and different molecular compositions of the deposits are dependent on the host tau genotypes injected with identical AD tau inoculums (Figure 9).

\section{Abbreviations}

AD: Alzheimer's disease

WT: wild type mouse expressing murine 4Rtau

P301S: transgenic mouse expressing human 4Rtau carrying the P301S mutation

mtWT: homozygous transgenic mouse knock-out for murine tau protein

hTau: homozygous transgenic mouse knock-out for murine tau protein and heterozygous expressing human forms of 3Rtau and 4Rtau proteins

3Rtau: tau with three repeats

4Rtau: tau with four repeats

AKT1: serine/threonine-protein kinase AKT1

$\beta$-actin; CK1- $\delta$ : casein kinase $\delta$

elF2a: eukaryotic initiation factor $2 a$

GSK $\beta$ : glycogen synthase kinase $3 \beta$

IRE1: inositol-requiring enzyme 1a

LAMP1: lysosomal-associated membrane protein 3

LC3: microtubule-associated protein 1A/1B-light chain 3

MAP2: microtubule-associated protein 2

NFL: neurofilament light molecular weight

p38: p38-kinase 
PHF1: anti-paired helical filament 1

PKAa/ $\beta$ : cAMP-dependent protein kinase A

SAPK/JNK: stress-activated protein kinase/Jun amino-terminal kinase

SRC: tyrosine-protein kinase SRC

Tau 5: monoclonal recognizing total tau protein

Tau AT8

Tau C3: tau truncated at aspartic acid 421

Tau MC1: conformation tau

Tau-N: nitrated tau

Tau-P phosphorylated tau. The phosphorylation or nitration of the protein at the specific site is indicated in every case

\section{Declarations}

\section{Ethical approval and consent to participate}

Brain samples of the hippocampus were obtained from the Institute of Neuropathology Brain Bank, Bellvitge University Hospital, following the guidelines of the Spanish legislation on this matter (Real Decreto Biobancos 1716/2011), and the approval of the local ethics committee of the Bellvitge University Hospital (Hospitalet de Llobregat, Barcelona, Spain). All animal procedures were carried out following the guidelines of the European Communities Council Directive 2010/63/EU and with the approval of the local ethical committee (C.E.E.A: Comitè Ètic d'Experimentació Animal; University of Barcelona, Spain; ref. 426/18).

The article is original, has not already been published in a journal, and is not currently under consideration by another journal

\section{Consent for publication}

All authors of the manuscript have read and agreed to its content and are accountable for all aspects of the accuracy and integrity of the manuscript in accordance with ICMJE criteria We agree to the terms of the BioMed Central Copyright and License Agreement, and, where applicable, Open Data policy.

\section{Availability of supporting data}

all the supporting data are in the manuscript 


\section{Competing interests}

No competing interests

\section{Funding}

The project leading to these results has received funding from "La Caixa" Banking Foundation under the project HR18-00452 to IF; it was also supported by the Ministry of Economy and Competitiveness, Institute of Health Carlos III (ISCIII) (co-funded by European Regional Development Fund, ERDF, a way to build Europe): FISPI17/000809 to IF; and the Intra-CIBERNED 2019 collaborative project to IF and JADR. We thank CERCA Programme/Generalitat de Catalunya for institutional support.

\section{Author's contribution}

PA-B prepared the colonies, inoculated the animals, prepared the inocula, processed the samples for morphological and biochemical studies, and checked the modifications in the samples; $\mathrm{MC}$ and $\mathrm{MJ}$ aided in the processing of the samples; JAR and IF designed the study; IF directed all the studies, analyzed the results, and prepared the text and figures. All the authors read and approved the final manuscript.

\section{Acknowledgements}

We wish to thank Benjamin Torrejón-Escribano (Advanced Light Microscopy Unit, Campus de Bellvitge, Scientific and Technical Facility, University of Barcelona), and Joana Cladera for their technical support. We are grateful to T. Yohannan for editorial assistance.

\section{References}

1. Kovacs GG. Invited review: Neuropathology of tauopathies: principles and practice. Neuropathol Appl Neurobiol 2015;41:3-23. doi: 10.1111/nan.12208.

2. Iqbal K, Liu F, Gong CX. Tau and neurodegenerative disease: the story so far. Nat Rev Neurol 2016;12:15-27. doi: 10.1038/nrneurol.2015.225.

3. Arendt T, Stieler JT, Holzer M. Tau and tauopathies. Brain Res Bull 2016;126: 238-292. doi: 10.1016/j.brainresbull.2016.08.018.

4. Lewis J, Dickson DW. Propagation of tau pathology: hypotheses, discoveries, and yet unresolved questions from experimental and human brain studies. Acta Neuropathol 2016;131:27-48. doi: 10.1007/s00401-015-1507-z.

5. Goedert M, Eisenberg DS, Crowther RA. Propagation of tau aggregates and neurodegeneration Annu Rev Neurosci 2017; 40:189-210. doi: 10.1146/annurev-neuro-072116-031153.

6. Iba M, Guo JL, McBride JD, Zhang B, Trojanowski JQ, Lee VM. Synthetic tau fibrils mediate transmission of neurofibrillary tangles in a transgenic mouse model of Alzheimer's-like tauopathy. $J$ Neurosci 2013; 33: 1024-1037. 
7. Peeraer E, Bottelbergs A, Van Kolen K, Stancu IC, Vasconcelos B, Mahieu M, Duytschaever H, Ver Donck L, Torremans A, Sluydts E, Van Acker N, Kemp JA, Mercken M, Brunden KR, Trojanowski JQ, Dewachter I, Lee VM, Moechars D. Intracerebral injection of preformed synthetic fibrils initiates widesprad tauopathy and neuronal loss in the brains of tau transgenic mice. Neurobiol Dis 2015;73:83-95. doi: 10.1016/j.nbd.2014.08.032.

8. Ahmed Z, Cooper J, Murray TK, Garn K, McNaughton E, Clarke H, Parhizkar S, Ward MA, Cavallini A, Jackson S, Bose S, Clavaguera F, Tolnay M, Lavenir I, Goedert M, Hutton ML, O'Neill MJ. A novel in vivo model of tau propagation with rapid and progressive neurofibrillary tangle pathology: the pattern of spread is determined by connectivity, not proximity. Acta Neuropathol 2014;127:667-683. doi: 10.1007/s00401-014-1254-6.

9. Clavaguera F, Bolmont T, Crowther RA, Abramowski D, Frank S, Probst A, Fraser G, Stalder AK, Beibel M, Staufenbiel M, Jucker M, Goedert M, Tolnay M. Transmission and spreading of tauopathy in transgenic mouse brain. Nat Cell Biol 2009; 111:909-913. doi: 10.1038/ncb1901.

10. Clavaguera F, Akatsu H, Fraser G, Crowther RA, Frank S, Hench J, Probst A, Winkler DT, Reichwald J, Staufenbiel M, Ghetti B, Goedert M, Tolnay M. Brain homogenates from human tauopathies induce tau inclusions in mouse brain. Proc Natl Acad Sci USA 2013; 110:9535-9540. doi: 10.1073/pnas. 1301175110 .

11. Clavaguera F, Hench J, Goedert M, Tolnay M. Invited review: prion-like transmission and spreading of tau pathology. Neuropathol Appl Neurobiol 2015;41:47-58. doi: 10.1111/nan.12197.

12. Boluda S, Iba M, Zhang B, Raible KM, Lee VM, Trojanowski JQ. Differential induction and spread of tau pathology in young PS19 tau transgenic mice following intracerebral injections of pathological tau from Alzheimer's disease or corticobasal degeneration brains. Acta Neuropathol 2015;129:221237 doi: 10.1007/s00401-014-1373-0.

13. Audouard E, Houben S, Masaracchia C, Yilmaz Z, Suain V, Authelet M, De Decker R, Buée L, Boom A, Leroy K, Ando K, Brion JP. High-molecular weight paired helical filaments from Alzheimer brain induces seeding of wild-type mouse tau into argyrophilic 4R tau pathology in vivo. Am J Pathol 2016; 186:2709-2722. doi: 10.1016/j.ajpath.2016.06.008.

14. Guo JL, Narasimhan S, Changolkar L, He Z, Stieber A, Zhang B, Gathagan RJ, Iba M, McBride JD, Trojanowski JQ, Lee VM. Unique pathological tau conformers from Alzheimer's brains transmit tau pathology in nontransgenic mice. J Exp Med 2016; 213:2635-2654. doi: 10.1084/jem.20160833

15. Narashima S, Guo JL, Changolkar L, Stieber A, McBride JD, Silva LV, He Z, Zhang B, Gathagan RJ, Trojanowski JQ, Lee VMY. Pathological tau strains from human brains recapitulate the diversity of tauopathies in non-transgenic mouse brain. J Neurosci 2017;37: 11406-11423. doi: 10.1523/JNEUROSCI.1230-17.2017.

16. Weitzman SA, Narasimhan S, He Z, Changolkar L, McBride JD, Zhang B, Schellenberg GD, Trojanowski, Lee VMY. Insoluble tau from human FTDP-17 cases exhibit unique transmission properties in vivo. J Neuropathol Exp Neurol 2020;79: 941-949. doi: 10.1093/jnen/nlaa086. 
17. Goedert M. Tau proteinopathies and the prion concept. Prog Mol Biol Transl Sci2020;175: 239-259. doi: 10.1016/bs.pmbts.2020.08.003.

18. Goedert M, Spillantini MG. Propagation of tau aggregates. Mol Brain 2017; 10:18. doi: 10.1186/s13041-017-0298-7

19. Mudner A, Colin M, Dujardin S, Medina M, Dewachter I, Naini SMA, Mandelkow EM, Mandelkow E, Luc Buée L, Michel Goedert M, Brion JP. What is the evidence that tau pathology spreads through prion-like propagation? Acta Neuropathol Commun 2017; 5:99. doi :1186/s40478-017-0488-7

20. Vaquer-Alicea J, Diamond MI, Joachimiak LA. Tau strains shape disease. Acta Neuropathologica (2021) 142:57-71. https://doi.org/10.1007/s00401-021-02301-7

21. Tseng JH, Ajit A, Tabassum Z, Patel N, Tian X, Chen Y, Prevatte AW, Ling K, Rigo F, Meeker RB, Herring LE, Cohen TJ. Tau seeds are subject to aberrant modifications resulting in distinct signatures. Cell Rep 2021;35:109037. doi: 10.1016/j.celrep.2021.109037.

22. Ferrer I, Aguiló García M, López González I, Diaz Lucena D, Roig Villalonga A, Carmona M, Llorens F, Garcia-Esparcia P, Martinez-Maldonado A, Frau Mendez M, Torrejón Escribano B,Bech-Serra JJ, Sabido E, de la Torre Gómez C,Del Rio JA. Aging-related tau astrogliopathy (ARTAG): not only tau phosphorylation in astrocytes. Brain Pathol 2018; 28: 965-985. doi: 10.1111/bpa.12593. E

23. Ferrer I, Aguiló García M, Carmona M, Andrés-Benito P, Torrejón-Escribano B, Garcia-Esparcia P, del Rio JA. Involvement of oligodendrocytes in tau seeding and spreading in tauopathies Front Aging Neurosci 2019; 11:112. doi: 10.3389/fnagi.2019.00112.

24. Ferrer I, Andrés-Benito P, Sala-Jarque J, Gil V, Del Rio JA. Capacity for seeding and spreading of argyrophilic grain disease in a wild-type murine model; comparisons with primary age-related tauopathy. Front Mol Neurosci 2020a; 13:101. doi: 10.3389/fnmol.2020.00101.

25. Ferrer I, Andrés-Benito P, Zelaya MV, Aguirre MEE, Carmona M, Ausín K, Lachén-Montes M, FernándezIrigoyen J, Santamaría E, Del Rio JA. Familial globular glial tauopathy linked to MAPT mutations: molecular neuropathology and seeding capacity of a prototypical mixed neuronal and glial tauopathy. Acta Neuropathol 2020b;139:735-771. doi: 10.1007/s00401-019-02122-9.

26. Ferrer I, Zelaya MV, Aguiló García M, Carmona M, López-González I, Andrés-Benito P López-González I, Andrés-Benito P, Lidón L, Gavín R, Garcia-Esparcia P, Del Rio JA. Relevance of host tau in tau seeding and spreading in tauopathies. Brain Pathol 2020c; 30 :298-318. doi: 10.1111/bpa.12778.

27. Ferrer I. Brain banking. In: Aminoff MJ, Daroff RB, eds. Encyclopedia of the Neurological Sciences, 2nd ed. Vol. 1. Oxford: Academic Press; 2014;467-473

28. López-González I, Aso E, Carmona M, Armand-Ugon M, Blanco R, Naudí A, Cabré R, Portero-Otin M, Pamplona R, Ferrer I. Neuroinflammatory gene regulation, mitochondrial function, oxidative stress, and brain lipid modifications with disease progression in tau P301S transgenic mice as a model of frontotemporal lobar degeneration-tau. J Neuropathol Exp Neurol 2015;74: 975-999. doi: 10.1097/NEN.0000000000000241.

29. Pitera AP, Asuni AA, O'Connor V, Deinhardt K. Pathogenic tau does not drive activation of the unfolded protein response J Biol Chem 2019; 294: 9679-9688. Doi: 10.1074/jbc.RA119.008263 
30. Andorfer C, Kress Y, de Silva SM, Tucker KL, Barde YA, Duff K, Davies P. Hyperphosphorylation and aggregation of tau in mice expressing normal human tau isoforms. J Neurochem 2003; 86: 582-590. doi: 10.1046/j.1471-4159.2003.01879.x.

31. Reynolds MR, Reyes JF,1 Fu Y, Bigio EH, Guillozet-Bongaarts AL, Berry RW, Binder LI. Tau nitration occurs at tyrosine 29 in the fibrillar lesions of Alzheimer's disease and other tauopathies. $J$ Neurosci 2006; 26:1036-1045. doi: 10.1523/JNEUROSCI.2143-06.2006.

32. Hitt BD, Vaquer-Alicea J, Manon VA, Beaver JD, Kashmer OM, Garcia JN, Diamond MI. Ultrasensitive tau biosensor cells detect no seeding in Alzheimer's disease. CSF Acta Neuropathol Commun 2021;9:99.doi: 10.1186/s40478-021-01185-8.

33. LoPresti P, Szuchet S, Papasozomenos SC, Zinkowski RP, Binder LI. Functional implications for the microtubule-associated protein tau: localization in oligodendrocytes. Proc Natl Acad Sci USA 1995; 92: 10369-10373. doi: 10.1073/pnas.92.22.10369.

34. Gorath M, Stahnke T, Mronga T, Goldbaum O, Richter-Landsberg C. Developmental changes of tau protein and mRNA in cultured rat brain oligodendrocytes. Glia 2001; 36: 89-101. doi: 10.1002/glia.1098.

35. Zareba-Paslawska J, Patra K, Kluzer L, Revesz T, Svenningsson P. Tau isoform-driven CBD pathology transmission in oligodendrocytes in humanized tau mice. Front Neurol 2021;11:589471.doi: 10.3389/fneur.2020.589471.

36. Funk KE, Mrak RE, Kuret J. Granulovacuolar degeneration (GVD) bodies of Alzheimer's disease (AD) resemble late-stage autophagic organelles. Neuropathol Appl Neurobiol 2011; 37:295-306.

37. Köhler C (2016) Granulovacuolar degeneration: a neurodegenerative change that accompanies tau pathology. Acta Neuropathologica 2016;132: 339-359. doi: 10.1007/s00401-016-1562-0.

38. Hoozemans JJ, van Haastert ES, Nijholt DA, Rozemuller AJ, Eikelenboom P, Scheper W. The unfolded protein response is activated in pretangle neurons in Alzheimer's disease hippocampus. Am J Pathol 2009;174:1241-1251. doi: 10.2353/ajpath.2009.080814.

39. Wiersma VI, van Ziel AM, Vazquez-Sanchez S, Nölle A, Berenjeno-Correa E, Bonaterra-Pastra A, Clavaguera F, Tolnay M, Musters RJP, van Weering JRT, Verhage M, Hoozemans JJM, Scheper W (2019) Granulovacuolar degeneration bodies are neuron-selective lysosomal structures induced by intracellular tau pathology. Acta Neuropathologica 138:943-970. doi: 10.1007/s00401-019-02046-4.

40. Liu M, Sui D, Dexheimer T, Hovde S, Deng X, Wang KW, Lin HL, Chien HT, Kweon HK, Kuo NS, Ayoub CA, Jimenez-Harrison D, Andrews PC, Kwok R, Bochar DA, Kuret J, Fortin J, Tsay YG, Kuo MH. Hyperphosphorylation renders tau prone to aggregate and to cause cell death. Mol Neurobiol 2020;57:4704-4719. doi: 10.1007/s12035-020-02034-w.

41. Villa González M, Vallés-Saiz L, Hernández IH, Avila J, Hernández F, Pérez-Alvarez MJ. Focal cerebral ischemia induces changes in oligodendrocytic tau isoforms in the damaged area. Glia2020;68:24712485.doi: $10.1002 /$ glia.23865.

42. Janke C, Beck M, Stahl T, Holzer M, Brauer K, Bigl V, Arendt T. Phylogenetic diversity of the expression of the microtubule-associated protein tau: implications for neurodegenerative disorders. Brain Res 
Mol Brain Res 1999;68:119-28.doi: 10.1016/s0169-328x(99)00079-0.

43. Hefti MM, Farrell K, Kim S, Bowles KR, Fowkes ME, Raj T, Crary JF. High-resolution temporal and regional mapping of MAPT expression and splicing in human brain development. PLoS One 2018; 13:e0195771. doi: 10.1371/journal.pone.0195771.

44. Qian W, Liu F. Review· Regulation of alternative splicing of tau exon 10. Neurosci Bull 2014; 30: 367377. doi: 10.1007/s12264-013-1411-2.

45. Duncan PI, Stojdl DF, Marius RM, Scheit KH, Bell JC. The Clk2 and Clk3 dual-specificity protein kinases regulate the intranuclear distribution of SR proteins and influence premRNA splicing. Exp Cell Res 1998; 241: 300-308. doi: 10.1006/excr.1998.4083.

46. Mermoud JE, Cohen PT, Lamond Al. Regulation of mammalian spliceosome assembly by a protein phosphorylation mechanism. EMBO J 1994; 13: 5679-5688. PMID: 7988565

47. Stojdl DF, Bell JC. SR protein kinases: the splice of life. Biochem Cell Biol 1999; 77: 293-298. PMID: 10546892

48. Hernandez F, Perez M, Lucas JJ, Mata AM, Bhat R, Avila J. Glycogen synthase kinase-3 plays a crucial role in tau exon 10 splicing and intranuclear distribution of SC35. Implications 376 Neurosci Bull April 1, 2014, 30(2): 367-377 for Alzheimer's disease. J Biol Chem 2004; 279: 3801-3806. doi: $10.1074 / j b c . M 311512200$

49. Kvissel AK, Orstavik S, Eikvar S, Brede G, Jahnsen T, Collas P, Göran Akusjärvi, Bjørn Steen Skålhegg. Involvement of the catalytic subunit of protein kinase A and of HA95 in pre-mRNA splicing. Exp Cell Res 2007; 313: 2795-2809. doi: 10.1016/j.yexcr.2007.05.014.

50. Patel NA, Kaneko S, Apostolatos HS, Bae SS, Watson JE, Davidowitz K, David S Chappell, Morris J Birnbaum, Jin Q Cheng, Denise R Cooper. Molecular and genetic studies imply Akt-mediated signaling promotes protein kinase Cbetall alternative splicing via phosphorylation of serine/argininerich splicing factor SRp40. J Biol Chem 2005 ; 280: 14302-14309. doi: 10.1074/jbc.M411485200.

51. Shi J, Qian W, Yin X, Iqbal K, Grundke-lqbal I, Gu X, Ding F, Gong CX, Liu F. Cyclic AMP-dependent protein kinase regulates the alternative splicing of tau exon 10: a mechanism involved in tau pathology of Alzheimer's disease. J Biol Chem 2011; 286: 14639-14648. doi:

10.1074/jbc.M110.204453.

52. Dujardin S, Commins C, Lathuiliere A, Beerepoot P, Fernandes AR, Kamath TV, De Los Santos MB, Klickstein N, Corjuc DL, Corjuc BT, Dooley PM, Viode A, Oakley DH, Moore BD, Mullin K, Jean-Gilles D, Clark R, Atchison K, Moore R, Chibnik LB, Tanzi RE, Frosch MP, Serrano-Pozo A, Elwood F, Steen JA, Kennedy ME, Hyman BT. Tau molecular diversity contributes to clinical heterogeneity in Alzheimer's disease. Nat Med 2020;26:1256-1263. doi: 10.1038/s41591-020-0938-9.

53. Li L, Shi R, Gu J, Tung YC, Zhou Y, Zhou D, Wu R, Chu D, Jin N, Deng K, Xu J, Gong CX, Iqbal K, Liu F. Alzheimer's disease brain contains tau fractions with differential prion-like activities. Acta Neuropathol Commun 2021;9:28. doi: 10.1186/s40478-021-01127-4.

\section{Figures}




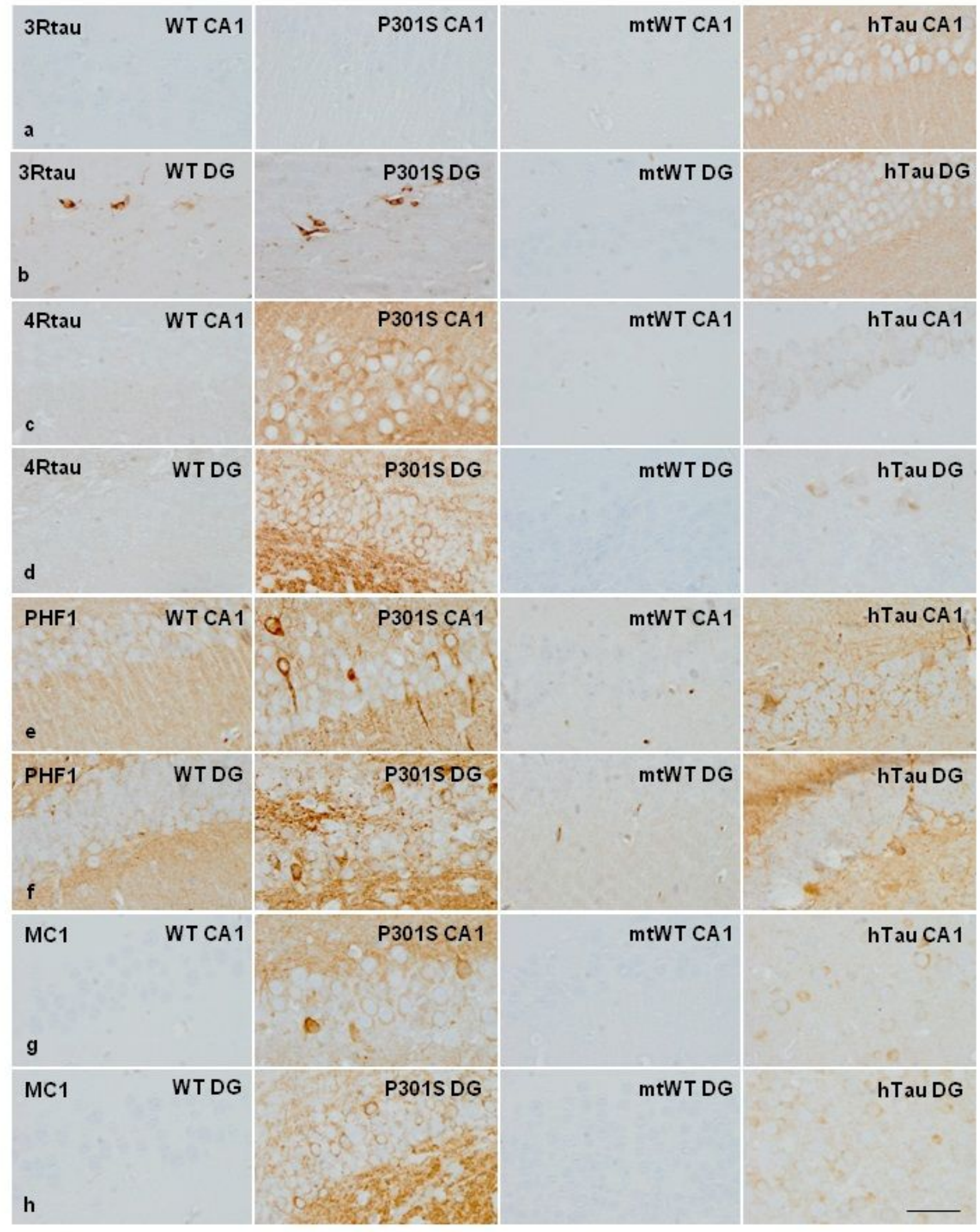

\section{Figure 1}

Main immunohistochemical characteristics of deposits in WT, P301S, mtWT and hTau. Representative images of 3Rtau $(a, b), 4 R t a u(c, d)$, PHF1 $(e, f)$ and $M C 1(g, h)$ in the CA1 region of the hippocampus (CA1) and dentate gyrus (GD) in WT, P301S, mtWT (mutant wild-type KO for murine tau, and hTau at the age of 9/10 months processed in parallel. 3Rtau predominates in hTau mice whereas only 4Rtau is expressed in P301S mice. For comparison, the expression of 3Rtau and 4Rtau is higher when compared 
with WT mice. Abnormal PHF1-positive neurons reminiscent of pre-tangles are found in P301S mice which are different from the weak PHF1-immunoreactive neuronal deposits in hTau mice. Weak PHF1 immunoreactivity is seen in the neuropil in WT mice (which is consistent with the observations in western blots). MC1 antibodies decorate pre-tangles in P301S but weak globular cytoplasmic deposits in hTau mice. MC1 is negative in WT. As expected antibodies against 3Rtau, 4Rtau, PhF1 and MC1 do not show the presence of tau in mtWT transgenic mice. Paraffin sections with slight haematoxylin counterstaining, bar $=50 \mu \mathrm{m}$.
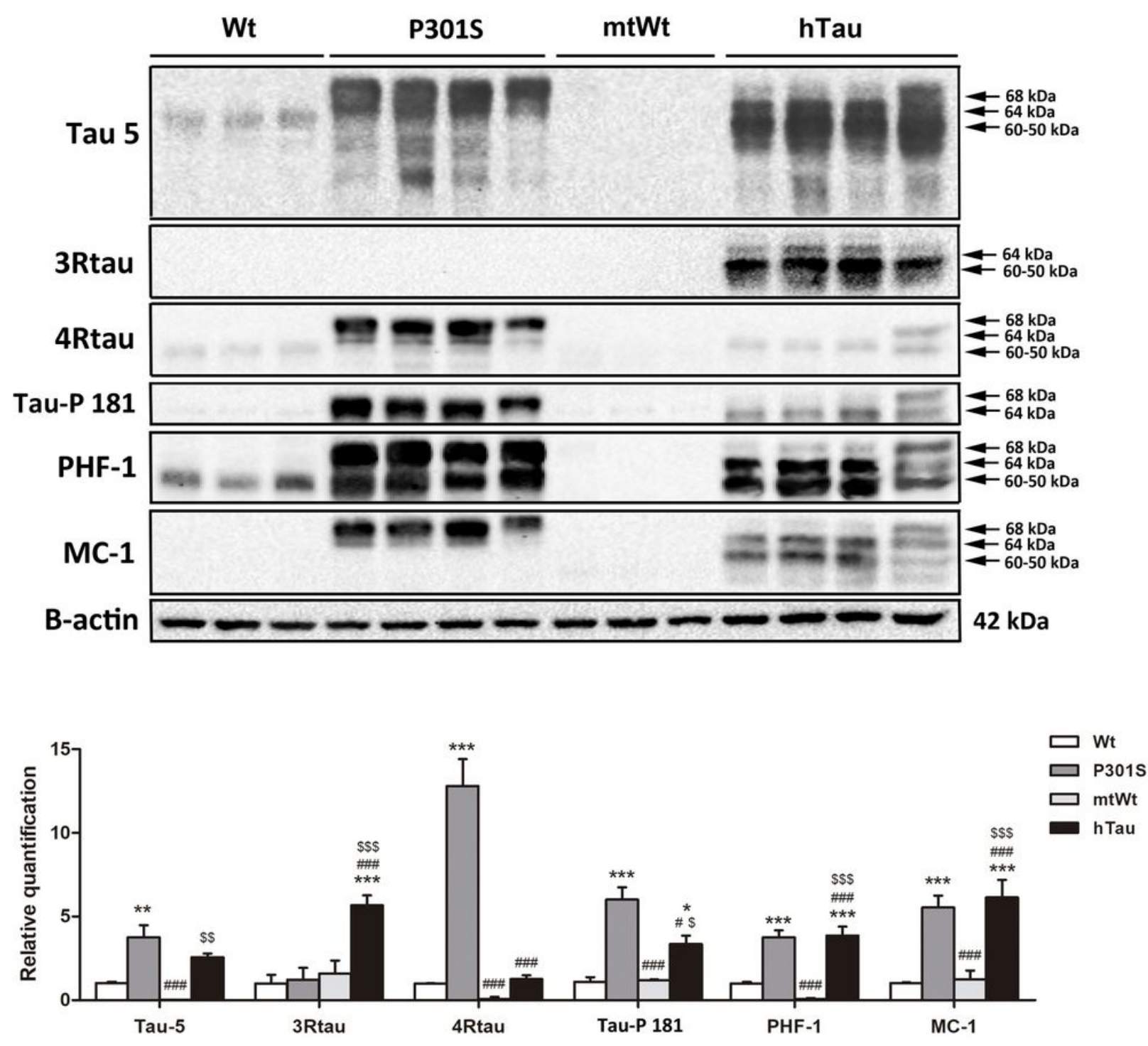

Figure 2

Biochemical characteristics of tau deposits in WT, P301S, mtWT and hTau. Gel electrophoresis and western blotting of total brain homogenates from wild type (WT), P301S, mutant wild-type KO for murine tau (mtWt), and hTau transgenic mice at the age of 9/10 months; blots were processed with Tau 5, 4Rtau, 
3Rtau, phosphorylated tau at Thr181 (tau-Pthr181), PHF1, and MC1. $\beta$-actin was used as a control of protein loading. Since the profile of $\beta$-actin was similar in the different western blots, only one representative example is included in the figure. P301S and hTau transgenic mice express much more tau than do WT mice. P301S expresses only 4Rtau whereas hTau expresses abundant 3Rtau and lesser amounts of 4 Rtau. In both transgenic mice, tau is phosphylated as revealed with tau-P Thyr181, PHF1, and MC1 antibodies, but the band pattern differs from one genotype to another. Two bands of high molecular weight characterize P301S mice whereas three/four bands of high and low molecular weight characterize hTau mice. As expected, $\mathrm{mtWt}$ mice lack tau of any species. The molecular weights labeling tau bands are approximate. The graph shows the relative quantification by western blotting of tau protein levels of tau proteins in total brain homogenate of WT, P301S, mtWt, and hTau mice aged 9/10 months. One-way analysis of variance (ANOVA) followed by Tukey post-test. Data are expressed as the mean values \pm SEM. The significance level was set at $* P<0.05 * * P<0.01$, ${ }^{\star * \star} P<0.001$ vs. WT; $\# P<0.05$, $\# \# \#$ P $<0.001$ vs. P301S; and $\$ \mathrm{P}<0.05$;

$$
P<0.01 \text { and }
$$

$\$ \mathrm{P}<0.001$ vs. mtWt.

\section{WT inocul}

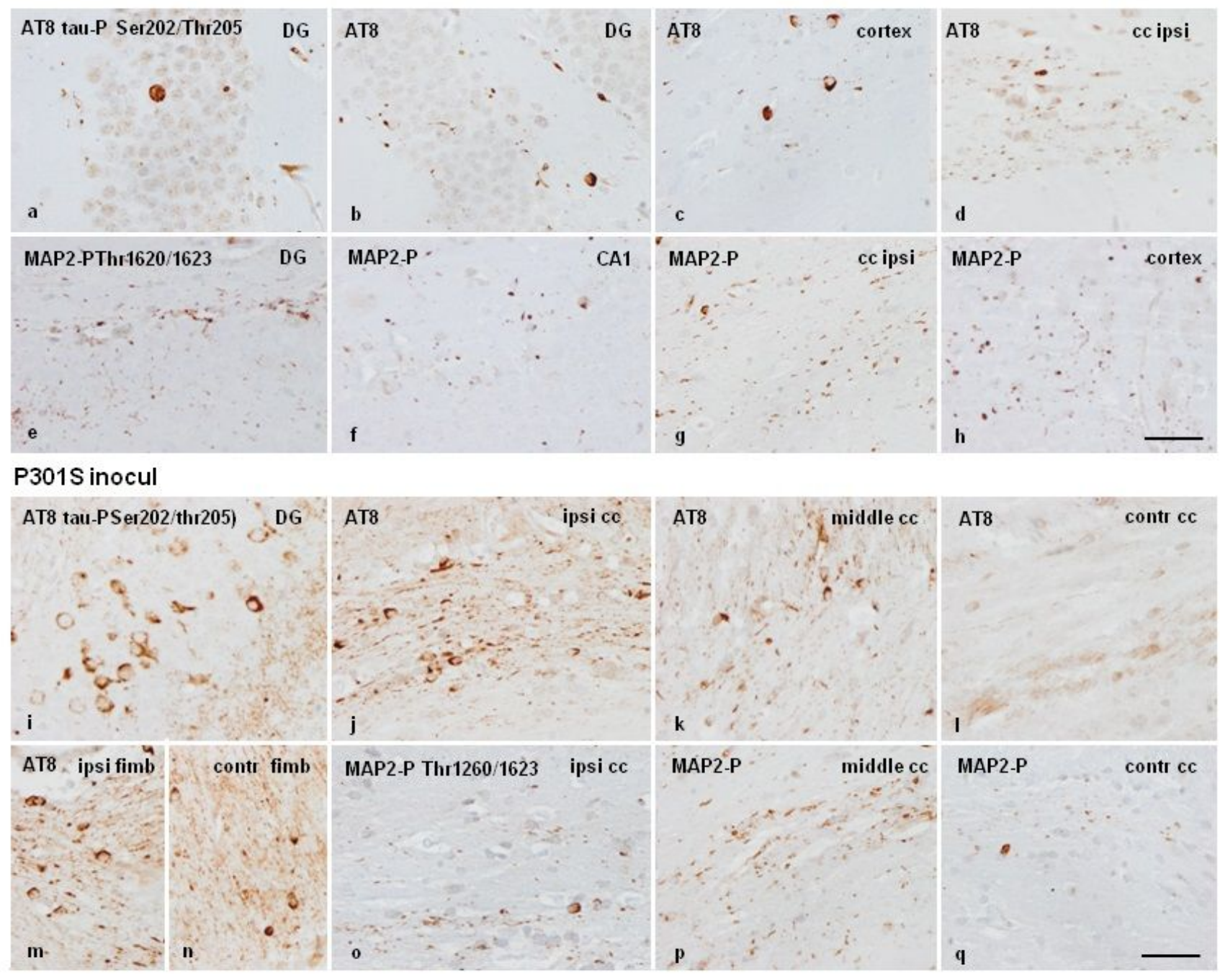




\section{Figure 3}

Inoculated WT and P301S mice. WT mice and P301S mice unilaterally inoculated in the hippocampus/dentate gyrus with sarkosyl-insoluble fractions from AD homogenates at the age of six months and killed at the age of nine months. In inoculated WT mice, AT8-immunoreactive neurons are depicted in the dentate gyrus, cerebral cortex, and ipsilateral corpus callosum (a-d). Abnormal deposits are also stained with anti-MAP2-P Thr1620/1623 antibodies including neurons, threads, and glial cells (eh). In inoculated P301S transgenic mice, AT8-immunoreactive deposits are abundant in neurons of the dentate gyrus (i); ipsilateral, middle region, and more sparse in the contralateral corpus callosum (j-1); ipsilateral and contralateral fimbria $(m, n)$. MAP2-P Thr1620/1623 antibodies also decorate abnormal inclusions in cells and threads (o-q). Note that the contralateral corpus callosum is variably affected; in some cases deposits are very rare or absent (I), but abnormal deposits spread to the contralateral corpus callosum in others (q). pDG: dentate gyrus; cc: corpus callosum; ipsi: ipsilateral; middle; contr: contralateral; CA1: CA1 region of the hippocampus; fimb: fimbria. Paraffin sections slightly counterstained with haematoxylin, bar $=50 \mu \mathrm{m}$.

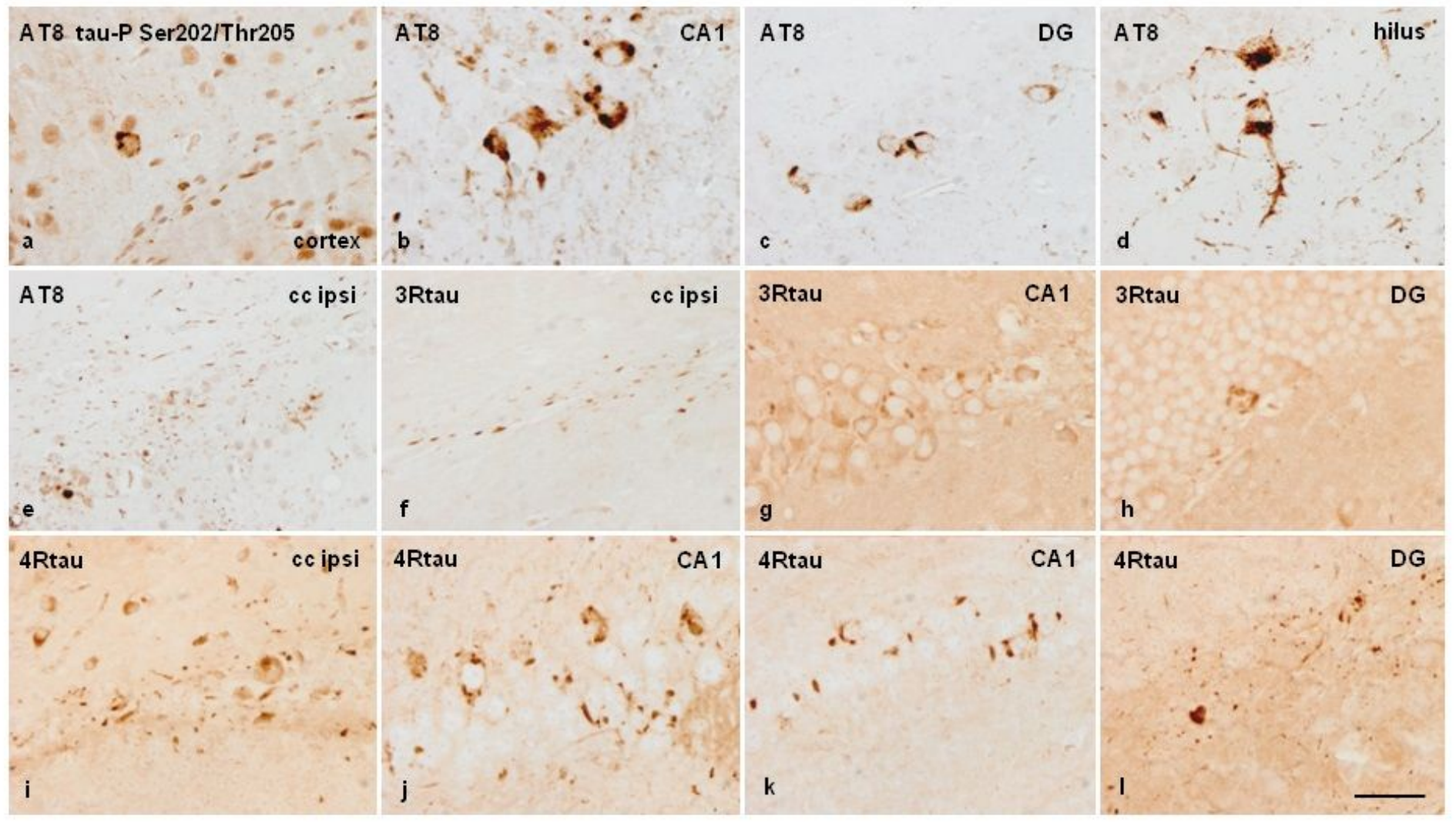

\section{Figure 4}

Inoculated hTau mice. hTau transgenic mice unilaterally inoculated in the hippocampus with sarkosylinsoluble fractions from $A D$ homogenates at the age of six months and killed at the age of nine months. AT8-immunoreactive deposits are seen in neurons of the cerebral cortex near the injection site, CA1 region of the hippocampus, dentate gyrus, and hilus (a-d); and in a few glial cells and threads in the corpus 
callosum (e). Tau deposits are stained with anti-3Rtau antibodies $(f, g, h)$ but deposition of 4Rtau is predominant (i-I). DG: dentate gyrus; cc: corpus callosum; ipsi: ipsilateral; CA1: CA1 region of the hippocampus. Paraffin sections slightly counterstained with haematoxylin, bar $=50 \mu \mathrm{m}$.

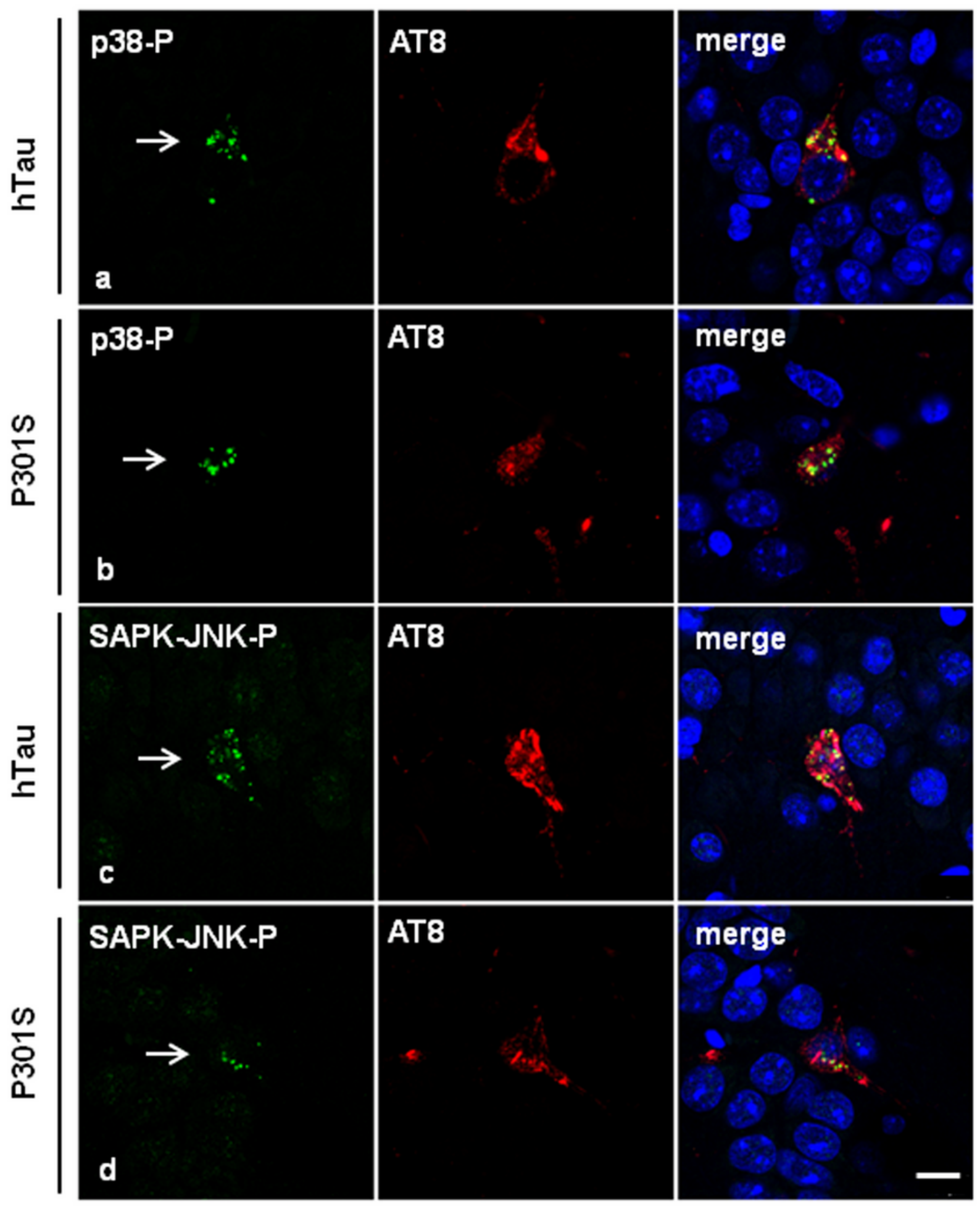

Figure 5

Active kinases in inoculated P301S and hTau mice. Double-labeling immunofluorescence and confocal microscopy to p38-P Thr180/Tyr182 or SAPK/JNK-PThr183/Thr185 (green), and AT8 (red) in inoculated 
and hTau $(a, c)$ and P301S (b, d) transgenic mice at the age of six months and killed at the age of nine months. Both active kinases (white arrows) co-localize tau deposits in granule cells of the dentate gyrus. Paraffin sections, nuclei are stained with DRAQ5TM (blue), bar $=10 \mu \mathrm{m}$.

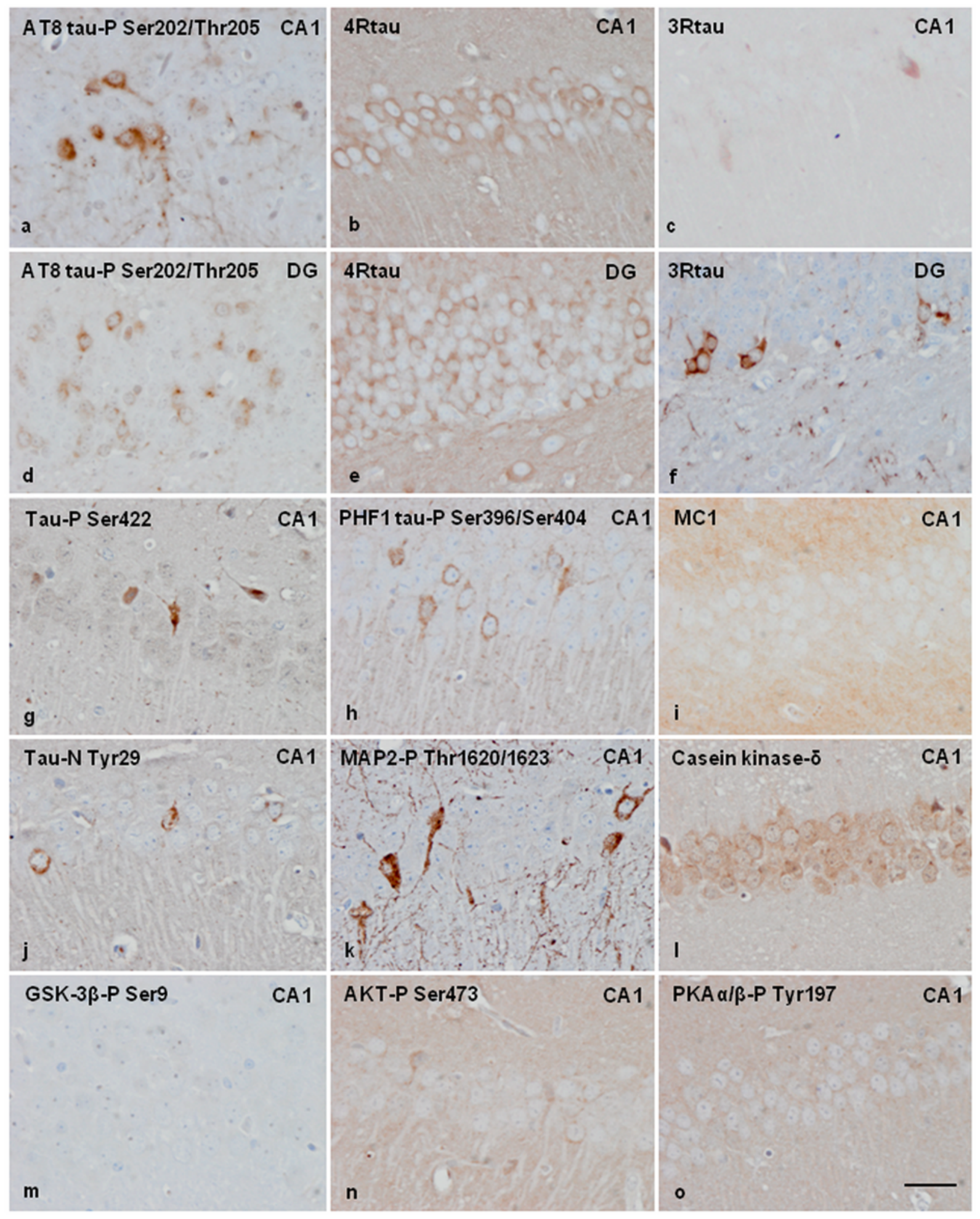

\section{Figure 6}

Regional characteristics of depois in inoculated P301S mice. P301S transgenic mice unilaterally inoculated with AD-tau at the age of three months and killed at the age of nine months. Inoculated P301S 
transgenic mice show phospho-tau deposition in neurons of the CA1 region of the hippocampus and neurons of the dentate gyrus, as revealed with the AT8 antibody (a, d). 4Rtau is not substantially increased in these regions, but 3Rtau is markedly augmented in a subpopulation of neurons of the dentate gyrus and mossy fibers (b, c, e, f). Note that the morphology of tau deposits is diffuse and decorates the cytoplasm and dendrites, resembling pre-tangles. Immunoreactivity to tau-P Ser422, PHF1, and MAP2-P is moderate or weak ( $\mathrm{g}, \mathrm{h}, \mathrm{k}$ ); $\mathrm{MC} 1$ negative, and tau-N Tyr29 negative or sparsely positive ( $\mathrm{i}$, j). CK1- $\delta$, GSK-3ß-P Ser9, AKT-P Ser473 and PKAa/ $\beta-P$ Tyr197 immunoreactivity is negative in neurons in inoculated P301S mice (I-o). Sections are consecutive and obtained from the same inoculated P301S mouse. Paraffin sections slightly counterstained with haematoxylin, bar $=50 \mu \mathrm{m}$. 


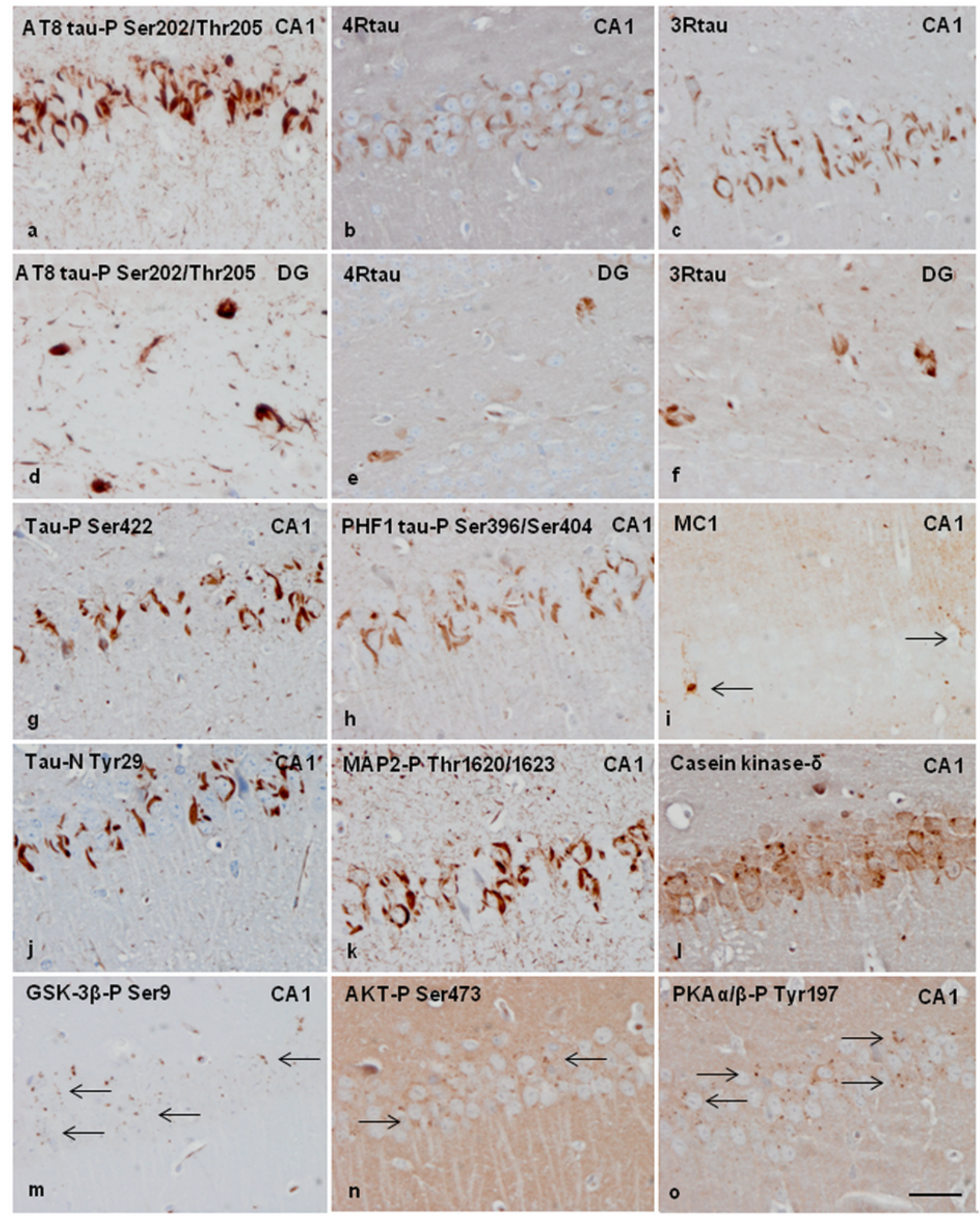

\section{Figure 7}

Regional characteristics of deposits in inoculated hTau mice. hTau transgenic mice unilaterally inoculated with AD-tau at the age of three months and killed at the age of nine months. Inoculated hTau transgenic mice show marked tau AT8 immunoreactivity in neurons of the CA1 region and neurons of the dentate gyrus $(a, d)$. Weak 4Rtau immunoreactivity is seen in affected neurons in these regions, but marked 3Rtau positivity is noted in CA1 and dentate gyrus neurons (b, c, e, f). Phospho-tau deposits in 
hTau transgenic mice are dense and flame-shaped, reminiscent of neurofibrillary tangles.

Immunoreactivity to tau-P Ser422, PHF1 MAP2-P and tau-N Tyr29 is strongly positive ( $g, h, j, k)$. MC1 immunoreactivity is also positive (i); in this particular field two neurons are depicted, one of them with small granular deposits (right arrow) and the other with a dense round inclusion (left arrow); other fields show images like those obtained with AT8 antibodies; this particular figure suggests progressive incorporation of conformational modifications in recruited tau deposit following inoculation. CK1-ס, GSK3ß-P Ser9, AKT-P Ser473 and PKAa/ $\beta-P$ Tyr197 granular immunoreactivity (arrows) appears in affected neurons in inoculated hTau mice (I-o). Sections are consecutive and obtained from the same inoculated hTau mouse. Paraffin sections slightly counterstained with haematoxylin, bar $=50 \mu \mathrm{m}$. 
Inoc hTau
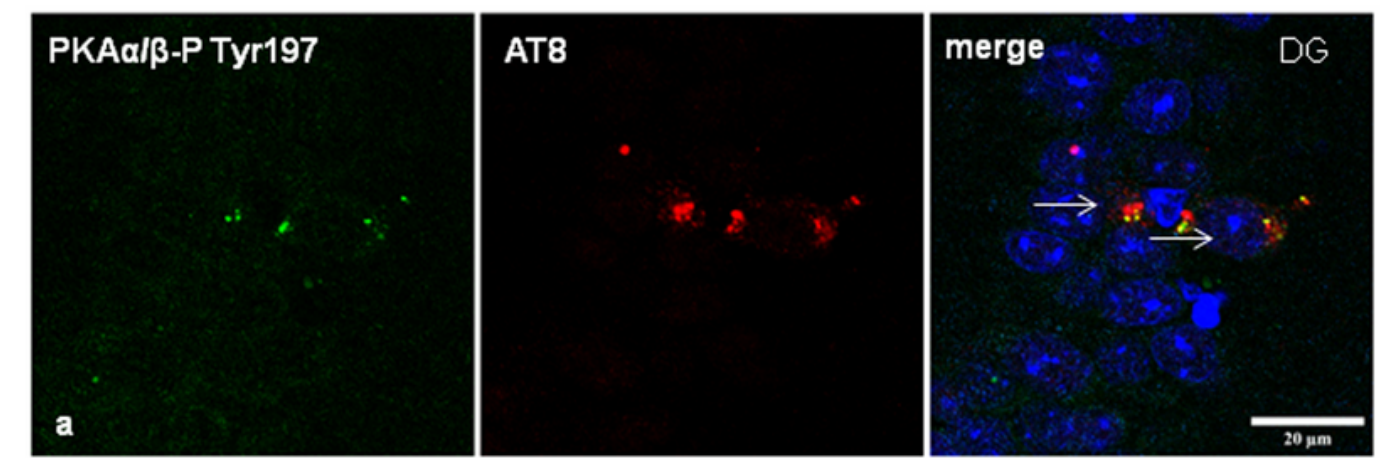

InochTau
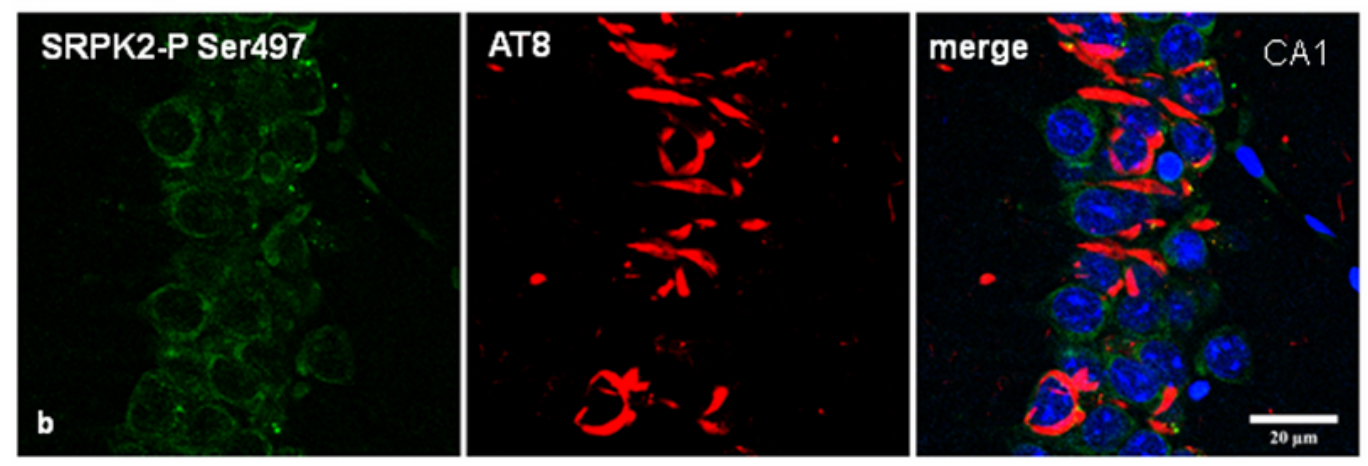

Inoc P301S
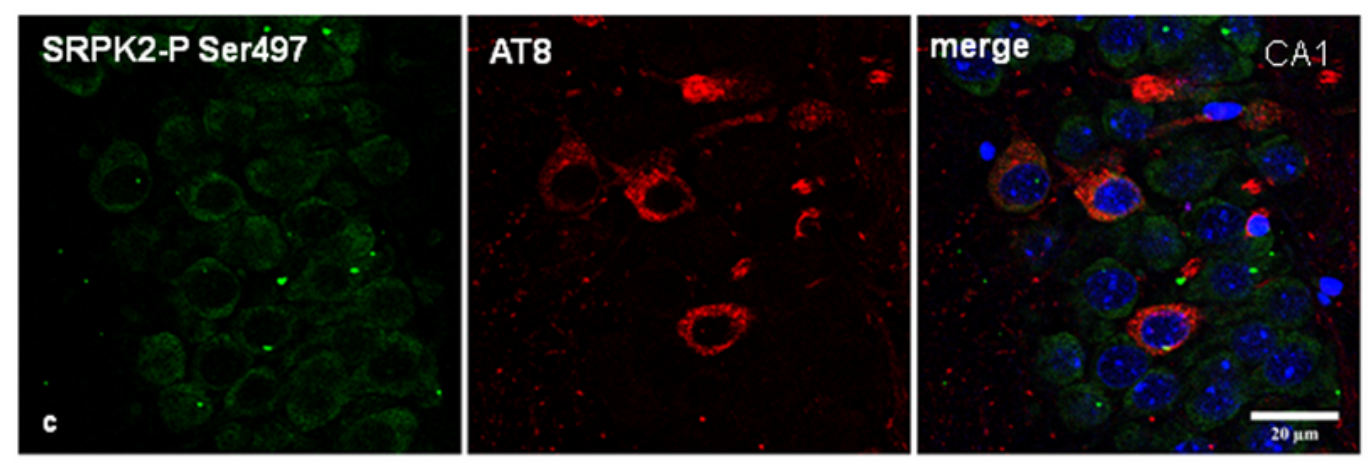

Inoc hTau

Inoc P301S

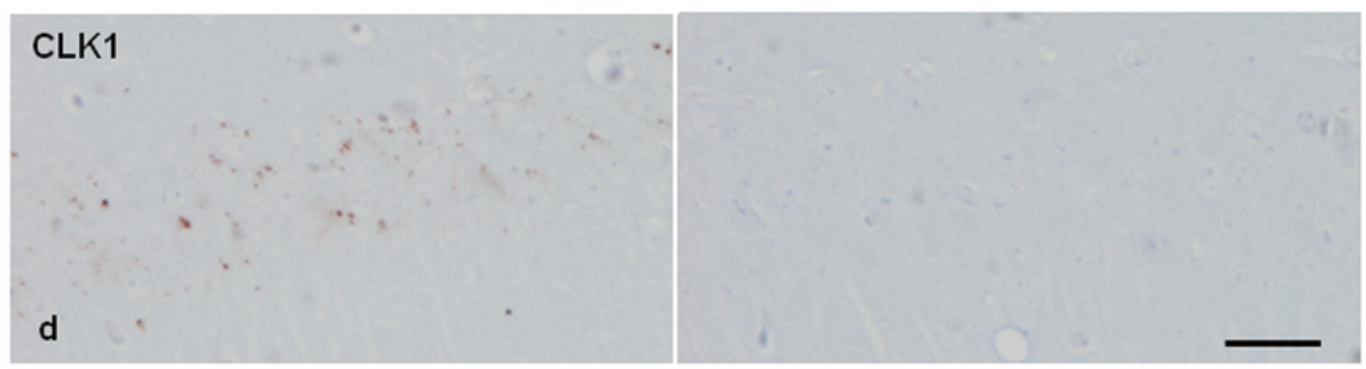

\section{Figure 8}

Expression of kinases involved in tau splicing Double-labelling immunofluorescence and confocal microscopy to PKAa/ $\beta-P$ Tyr197 and AT8 in inoculated hTau mice (a); SRPK2-P Ser497 and AT8 in inoculated hTau (b) and P301S (c) transgenic mice. Cytoplasmic granular PKAa/ $\beta-P$ Tyr197 immunoreactivity selectively co-localizes with abnormal phospho-tau deposits in neurons (arrows). In contrast, cytoplasmic SRPK2-Ser497 immunoreactivity is found equally in neurons with and without 
phosphor-tau deposits in hTau and P301S mice. Paraffin sections, nuclei stained with DRAQ5TM (blue), bar $=20 \mu \mathrm{m}$. (d) CLK1 immunoreactivity in the CA1 region of inoculated hTau and P301S mice. Small cytoplasmic granules are seen in inoculated hTau but not in inoculated P301S mice. Paraffin sections, slightly counterstained with haematoxylin, bar $=40 \mu \mathrm{m}$.

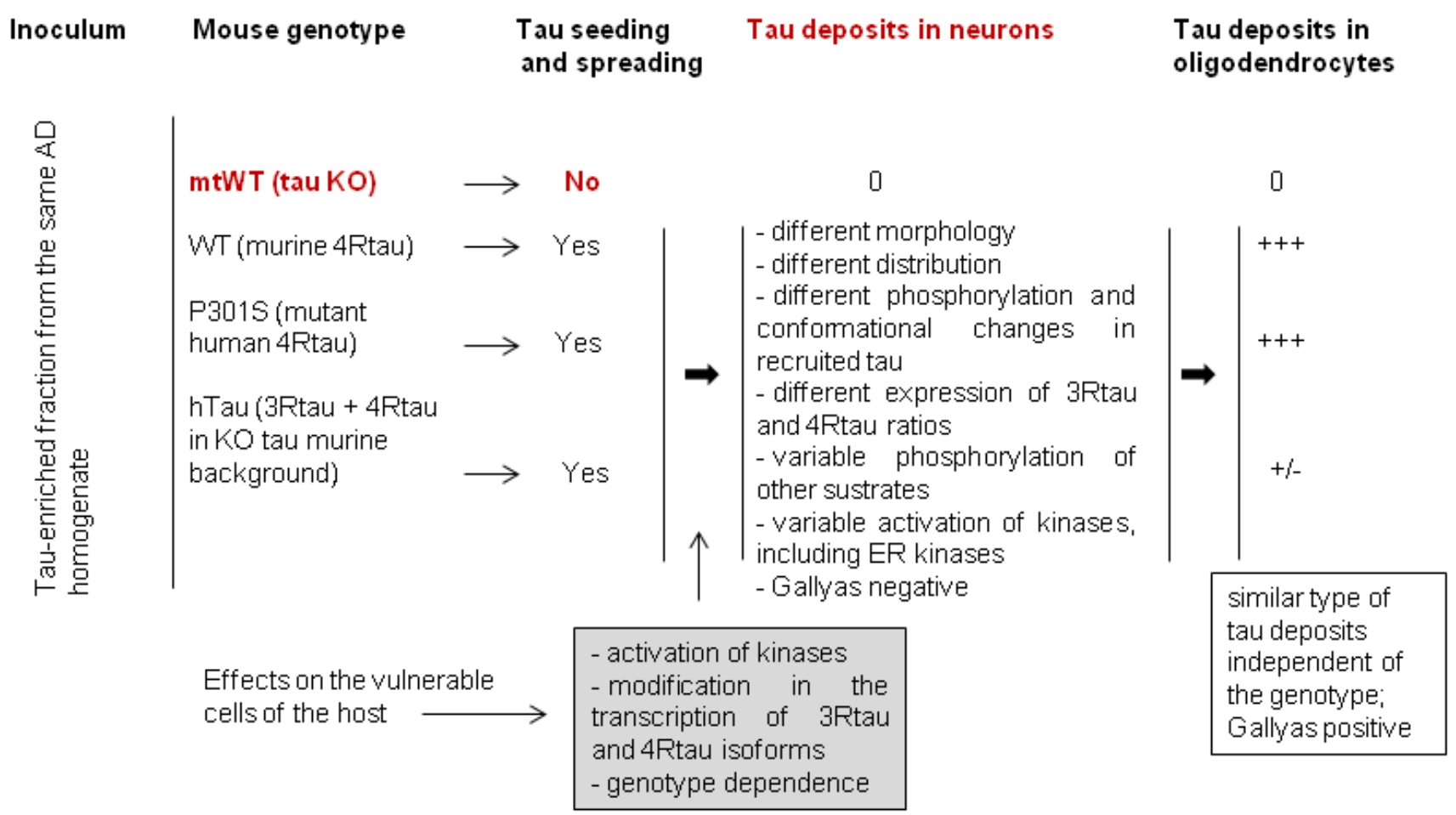

\section{Figure 9}

Graphic abstract

\section{Supplementary Files}

This is a list of supplementary files associated with this preprint. Click to download.

- SupplFig.1.tiff

- SupplFig.2.tif

- SupplFig.3.tif 
- SupplFig.4.tif

Page $41 / 41$ 